\title{
Evaluación de la organización académico administrativa con base en la metodología de los sistemas suaves: El caso de la licenciatura en turismo
}

\section{Evaluation of the Academic-administrative Organization based on the Soft Systems Methodology: the Case of the Degree in Tourism}

\author{
Edgar Oliver Cardoso Espinosa ${ }^{I}$ \\ Escuela Superior de Comercio y Administración (ESCA) \\ Instituto Politécnico Nacional \\ México \\ eoce@hotmail.com \\ José Roberto Ramos Mendoza ${ }^{2}$ \\ Escuela Superior de Comercio y Administración (ESCA) \\ Instituto Politécnico Nacional \\ México \\ dr.ramos.ipn@hotmail.com \\ María Trinidad Cerecedo Mercado ${ }^{3}$ \\ Escuela Superior de Comercio y Administración (ESCA) \\ Instituto Politécnico Nacional \\ México \\ tricermer@yahoo.com
}

Recibido 20 de octubre de 2010 • Aceptado 09 de marzo de 2011

\footnotetext{
Doctor en Ciencias Administrativas. Profesor-nvestigador de la Sección de Estudios de Posgrado e Investigación de la Escuela Superior de Comercio y Administración (ESCA) del Instituto Politécnico Nacional (IPN). Docente en la Maestría de Administración y Gestión de la Educación y el Doctorado de Ciencias Administrativas en el área de Evaluación de Programas Educativos e Institucionales.

2 Doctor en Ciencias Administrativas. Profesor-investigador de la Sección de Estudios de Posgrado e Investigación de la Escuela Superior de Comercio y Administración (ESCA) del Instituto Politécnico Nacional (IPN). Docente en la Maestría de Administración de Negocios en el área de Turismo.

3 Doctora en Ciencias Administrativas. Profesora-Investigadora de la Sección de Estudios de Posgrado e Investigación de la Escuela Superior de Comercio y Administración (ESCA) del Instituto Politécnico Nacional (IPN). Docente en la Maestría en Administración y Gestión de la Educación y el Doctorado en Ciencias Administrativas en el área de Organización y Dirección de Instituciones Educativas.
} 
Resumen. El objetivo de la investigación fue evaluar, con un enfoque sistémico, la organización académico administrativa de tres programas de licenciatura en turismo, con la finalidad de identificar las características que estos presentaban respecto a los criterios del Consejo para la Acreditación de la Educación Turística (CONAET) y TedQual (Tourism Education Quality), para acceder a la certificación nacional e internacional. El cuestionario y la guía de observación fueron los instrumentos para recopilar la información necesaria, la cual fue organizada con un enfoque cualitativo (mediante el Atlas Ti) y cuantitativo (a través de la estadística descriptiva). La principal conclusión fue que la Licenciatura en Turismo, ofrecida por la Escuela Superior de Turismo (EST), cumple con los criterios de certificación. Se considera, por tanto, que sus instalaciones y el programa ofertado se encuentran de acuerdo con los lineamientos de certificación, en tanto que la Universidad el Valle de México (UVM) y la Escuela Bancaria y Comercial (EBC) presentan serias deficiencias respecto a los criterios establecidos por estos organismos.

Palabras claves. Evaluación, sistemas suaves, turismo, organización.

Abstract. The goal of this study was to evaluate, through a systemic approach, the academicadministrative organization of three Bachelor's Degree Programs in Tourism, to identify their characteristics in reference to the criteria of the Consejo para la Acreditación de la Educación Turística (CONAET, by its Spanish acronym) and Tourism Education Quality (TedQual) for the national and international certification. The questionnaire and observation guide were the tools to gather the necessary information, which was organized through a qualitative (Atlas $\mathrm{Ti}$ ) and quantitative approach (descriptive statistics). The main conclusion was that the Bachelor's Degree offered by the Escuela Superior de Turismo (EST, by its Spanish acronym) meets the certification criteria (the program and facilities are under the certification guidelines). In contrast, Universidad el Valle de México (UVM, by its Spanish acronym) and the Escuela Bancaria y Comercial (EBC, by its Spanish acronym) have serious deficiencies according to the criteria established by the agencies.

Keywords. Evaluation, soft systems, tourism, organization.

\section{Introducción}

En la actualidad, el desarrollo de las naciones es de suma importancia, sobre todo por el mundo globalizado en el que se vive, lo cual requiere que los países posean, día a día, diferentes formas de enfrentar los retos de las actividades económicas mundiales que de estas emanan. El turismo no es la excepción, ya que demanda, en todo momento, acciones concretas por parte de los países respecto a diversificación de productos y servicios, desarrollo de nuevos complejos, entre otras; por lo que se requiere de la formación del recurso humano. Las instituciones de educación superior (IES), en coordinación con la gestión estratégica de la escuela, deben adaptarse y constantemente actualizarse en cuanto a sus cuerpos docentes, instalaciones, equipamientos y organización institucional.

Por su parte, la metodología de sistemas blandos o suaves (SSM por sus siglas en inglés Soft Systems Methodology), de Checkland (2002), se utiliza para aplicar a los sistemas estructurados a situaciones asistémicas con un alto componente social y humano, como el educativo. Esta particularidad distingue a la SSM de otras metodologías que se ocupan de problemas duros enfocados a la tecnología. Zexian y Xuhui (2010, p. 143) señalan que: "Esta metodología está fundada en que una organización no existe como un ente independiente, sino que es parte de un proyecto desarrollado por un grupo de personas comprometidas en un diálogo". Así, los sistemas suaves se identifican 
porque le dan mayor importancia, tanto al ámbito social como a las vinculaciones que se generan entre este y a las organizaciones. De esta manera, el enfoque sistémico considera la organización como una entidad que se encuentra en constante interacción con su ambiente. Señala, además, la existencia de diversas relaciones entre sus componentes internos llamados subsistemas. De este modo, dicha perspectiva conceptualiza a la organización como un sistema sociotécnico abierto, integrado de varios subsistemas importantes: objetivos y valores, técnico, psicosocial, estructural y administrativo.

En este sentido, comenta Barranco (2002) que la SSM es un proceso que en la actualidad ha tomado mayor auge en el ámbito educativo, pues toma en cuenta a todos los involucrados en el problema por diagnosticar, llevando a cabo acciones holísticas desde un enfoque hermenéutico, cuyo término refiere a la interpretación desde la percepción empirista del sujeto interviniente.

Por ello, esta metodología se presenta como una alternativa dentro de los modelos evaluativos, cuyo fin es "identificar los elementos que conforman el programa educativo, recabando información de orden cualitativo y cuantitativo, para emitir juicios de valor que permitan proporcionar cambios necesarios y consecuentes en la evaluación de programas" (Cardoso, Ramos y Tejeida, 2009, p. 32).

Lo anterior es producto del renovado interés por atender las necesidades educativas de los alumnos, mediante el denominado proceso evaluativo de programas educativos, no solo a nivel nacional, sino también internacional. Así, la evaluación de programas educativos se orienta a determinar si los servicios prestados por la organización resultan necesarios, si se utilizan, si son suficientes, si se dan en los términos planificados, si ayudan dentro de un costo razonable o si, incluso, provocan efectos no deseados. Por tanto, existen diferentes intenciones a la hora de evaluar los programas: a) dar seguimiento al programa para saber si es eficaz o no, e introducir modificaciones en su diseño; b) identificar problemas que se suscitan en el desarrollo aplicativo de este; c) desarrollar programas pilotos para mejorar la eficacia de estos; d) identificar efectos diferenciales en distintas poblaciones; e) determinar la relevancia y la validez de los principios del programa; f) verificar el cumplimiento de los objetivos en el ámbito para el cual fueron creados y g) tomar decisiones adecuadas en cuanto a su función educativa.

\section{Metodología}

\section{Objetivo de la investigación}

Evaluar con enfoque sistémico la organización académica administrativa de tres programas de licenciatura en Turismo, con base en los criterios de certificación del CONAET y TedQual.

\section{Tipo de investigación}

Se trató de una investigación evaluativa, descriptiva y no experimental, ya que se enfocó a observar las situaciones y fenómenos tal y como se dieron en sus propios contextos, para posteriormente analizarlos con la finalidad de emitir juicios de valor. Asimismo, fue de tipo transversal, ya que los datos fueron recolectados en un solo momento en el tiempo, con el principal propósito de analizar las variables con sus interrelaciones en un tiempo específico. 


\section{Selección de la muestra}

La muestra del estudio fue de tipo dirigida o no probabilística, ya que el investigador seleccionó los tres programas de licenciatura en Turismo, impartidos en tres IES y que fueron: a) Programa de Licenciados en Turismo (LT), impartido por la Escuela Superior de Turismo dependiente del Instituto Politécnico Nacional; b) Programa de Licenciado en Administración Hotelera y Turística (LAHT), impartido por la Escuela Bancaria y Comercial (Campus Reforma) y, c) Programa de Licenciado en Administración de Empresas Turísticas (LAET), impartido por la Universidad del Valle de México (Campus Chapultepec). Cabe señalar que en esta clase de muestra, "la elección de los elementos, no depende de la probabilidad sino de causas propias al investigador" (Hernández, Fernández y Baptista, 2002, p. 135).

\section{Diagrama de variables}

El diagrama de variables muestra la estrategia implementada en la investigación (figura 1)

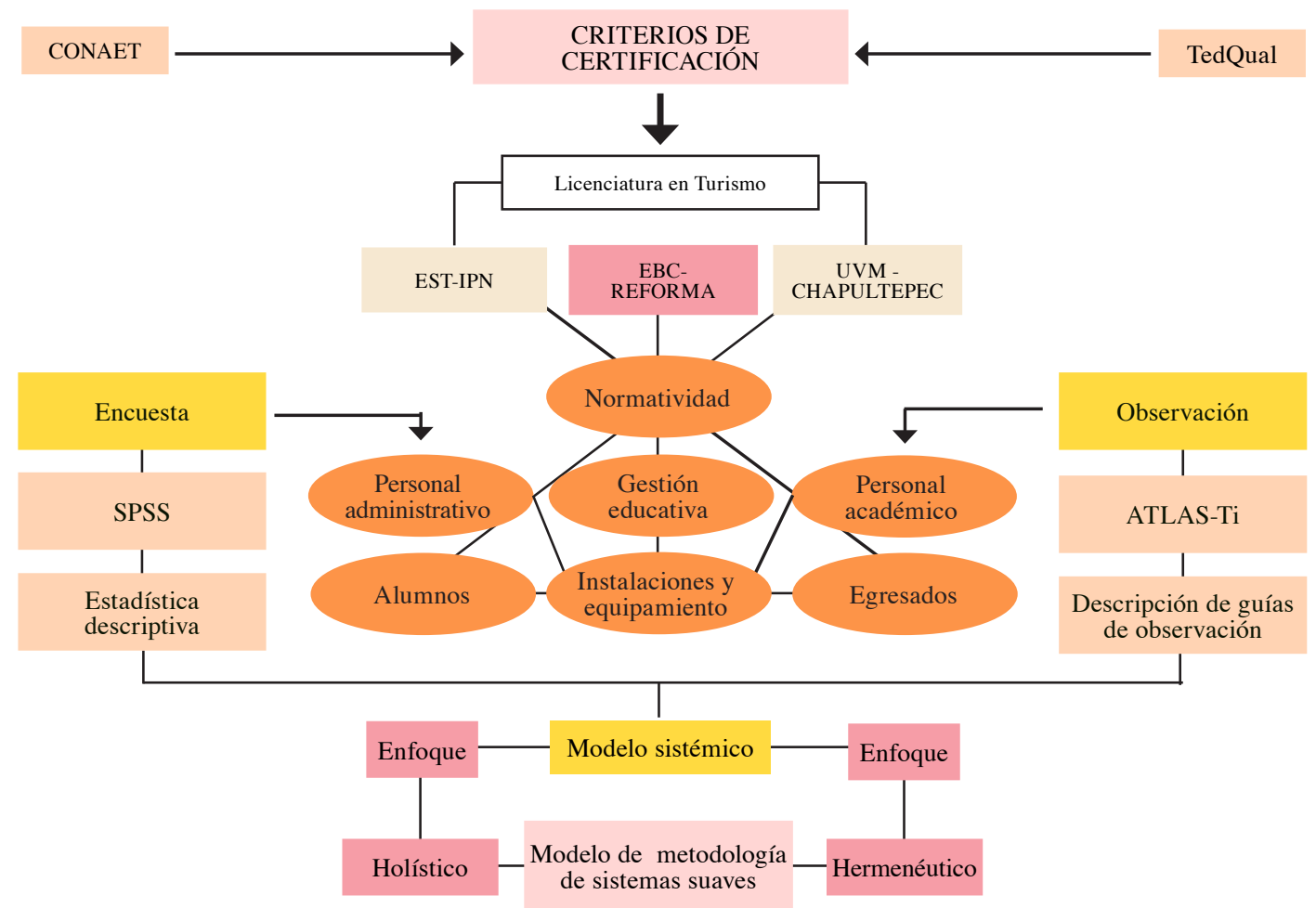

Figura 1. Diagrama de la investigación.

Nota: Elaboración propia. 


\section{Diseño de los instrumentos}

Los instrumentos utilizados fueron el cuestionario y la guía de observación (Tabla 1).

Tabla 1

Instrumentos para la investigación

\begin{tabular}{|c|l|}
\hline \multirow{2}{*}{ INSTRUMENTO } & \multicolumn{1}{c|}{ DESCRIPCIÓN } \\
\hline \multirow{7}{*}{ Cuestionario } & $\begin{array}{l}\text { Infraestructura: Permite la operación y desarrollo adecuado del programa educativo. } \\
\text { Está integrada por las aulas, talleres, laboratorios, centro de cómputo y biblioteca. }\end{array}$ \\
\cline { 2 - 3 } & $\begin{array}{l}\text { Estructura: Conjunto de actividades mediante las cuales se forma a un alumno, } \\
\text { en cuanto a su perfil de ingreso, permanencia y egreso, mismos que deben ser } \\
\text { congruentes con el fin del programa, y las condiciones imperantes en el sector } \\
\text { laboral. }\end{array}$ \\
\cline { 2 - 3 } & Supraestructura: Considera la misión, visión, concepciones y modelos educativos, \\
& perfiles de personal académico y alumnos. \\
\hline \multirow{7}{*}{ Guía de Observación } & $\begin{array}{l}\text { 1. Personal académico adscrito al programa. } \\
\text { 2. Currículo }\end{array}$ \\
& 3. Servicios institucionales de apoyo al aprendizaje de los estudiantes. \\
& 5. Gestión académico -administrativa. \\
& 6. Procesos de planeación y evaluación. \\
\hline
\end{tabular}

Nota: Elaboración propia.

\section{Análisis y resultados}

Con la finalidad de conformar las tablas para el análisis correspondiente, los resultados obtenidos a partir de los cuestionarios aplicados a alumnos, administrativos y docentes se organizaron con base en la estadística descriptiva para obtener las frecuencias. Además, se realizó el estudio de las variables mediante las guías de observación a las cuales se les aplicó el software de Atlas Ti.

\section{De los cuestionarios}

Con base en la tabla 2, la principal deficiencia de dos de las instituciones estudiadas y de carácter privado es la falta de investigación en tópicos referentes a la carrera que se imparte. En el caso de la UVM, presenta deficiencia en cuanto a las aulas con las que cuenta y que no se encuentran equipadas adecuadamente para su uso. Asimismo, tanto la UVM como la EBC presentan deficiencias en cuanto a los materiales de apoyo. Cabe señalar que, si la variable analizada se ubica por debajo de los estándares nacionales e internacionales, se evalúa como DEFICIENTE; en tanto que si se encuentra en un punto medio se valora como BUENO y hay que poner atención para superarlo y, si se encuentra dentro de los estándares nacionales e internacionales, se cataloga como EXCELENTE. 


\begin{tabular}{|c|c|c|c|c|c|c|c|c|c|c|c|c|c|c|c|c|c|c|c|c|c|c|c|c|}
\hline$\frac{\pi}{\tilde{Z}}$ & $\underset{I}{\underset{I}{x}}$ & 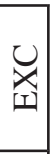 & 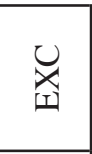 & & 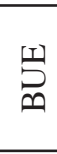 & 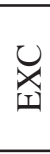 & $\begin{array}{l}\underset{x}{x} \\
\text {. }\end{array}$ & $\begin{array}{l}\frac{1}{T} \\
\vec{D}\end{array}$ & 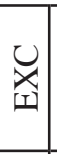 & $\begin{array}{l}u \\
x \\
x \\
y\end{array}$ & $\begin{array}{l}\frac{1}{1} \\
\frac{1}{D}\end{array}$ & & 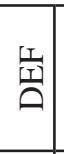 & $\begin{array}{l}\frac{1}{15} \\
\overrightarrow{0}\end{array}$ & $\begin{array}{l}\frac{1}{1} \\
\frac{1}{0}\end{array}$ & $\begin{array}{l}\text { 岦 } \\
\text { a }\end{array}$ & 峲 & $\begin{array}{l}\frac{1}{1} \\
\frac{1}{0}\end{array}$ & 党 & 勏 & 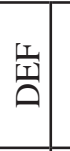 & $\begin{array}{l}\frac{\mathrm{I}}{\mathrm{I}} \\
\mathrm{D}\end{array}$ & 党 & $\begin{array}{l}\frac{1}{15} \\
\frac{1}{6}\end{array}$ \\
\hline 完 & $\begin{array}{c}\underset{x}{x} \\
\text { cy }\end{array}$ & $\begin{array}{l}u \\
\underset{I}{x} \\
\end{array}$ & 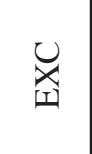 & & $\sum^{n}$ & $\underset{I}{x}$ & $\begin{array}{l}u \\
x \\
x\end{array}$ & $\begin{array}{l}\frac{1}{T} \\
\overrightarrow{0}\end{array}$ & 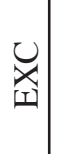 & $\underset{\text { 齐 }}{u}$ & 岂 & & $\begin{array}{l}\frac{1}{w} \\
\frac{1}{0}\end{array}$ & $\begin{array}{l}\frac{1}{15} \\
\frac{1}{0}\end{array}$ & $\begin{array}{l}\frac{1}{1} \\
\frac{1}{0}\end{array}$ & $\begin{array}{l}\text { 岦 } \\
\text { a }\end{array}$ & 耐 & 壱 & 壱 & 空 & 壹 & 吉 & $\frac{\mathrm{I}}{\mathrm{II}}$ & $\begin{array}{l}\frac{1}{15} \\
\text { 至 }\end{array}$ \\
\hline 品 & 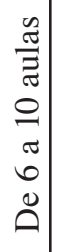 & 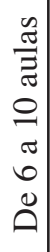 & 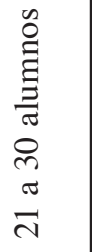 & & 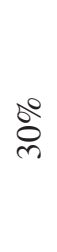 & 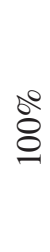 & $\begin{array}{l}80 \\
8 \\
0\end{array}$ & 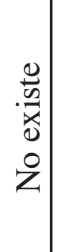 & $\begin{array}{l}\stackrel{0}{0} \\
8 \\
0\end{array}$ & $\begin{array}{l}\stackrel{0}{0} \\
8\end{array}$ & $\begin{array}{l}0 \\
\text { ô }\end{array}$ & & $\begin{array}{c}o \\
\vdots \\
\vdots \\
\vdots \\
\vdots\end{array}$ & 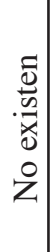 & 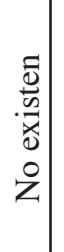 & 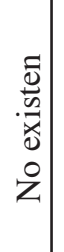 & 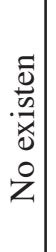 & 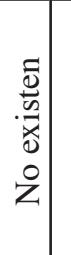 & 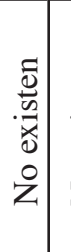 & 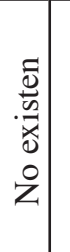 & 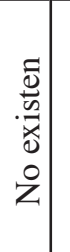 & 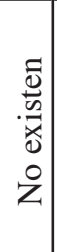 & 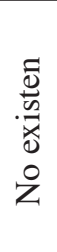 & 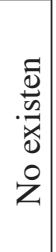 \\
\hline $\begin{array}{l}\frac{\pi}{\tilde{\sigma}} \\
\tilde{\sigma} \\
\tilde{\sigma}\end{array}$ & 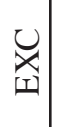 & $\begin{array}{l}\underset{x}{x} \\
\text { x }\end{array}$ & $\underset{I}{x}$ & & 齐 & $\begin{array}{l}\underset{x}{x} \\
\text {. }\end{array}$ & $\begin{array}{l}\underset{x}{x} \\
\text {. }\end{array}$ & 岂 & $\begin{array}{c}\underset{x}{x} \\
\text { cul }\end{array}$ & 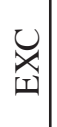 & $\stackrel{n}{\Sigma}$ & & $\underset{|=|}{\underset{I}{x}}$ & $\underset{\substack{x \\
\underset{I}{x}}}{u}$ & 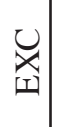 & 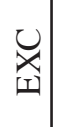 & $\begin{array}{l}\underset{x}{x} \\
\mid\end{array}$ & 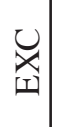 & $\begin{array}{l}\underset{x}{x} \\
\text {. }\end{array}$ & $\begin{array}{l}\underset{x}{x} \\
. \\
\mid\end{array}$ & $\begin{array}{l}\underset{x}{x} \\
.\end{array}$ & $\begin{array}{l}\underset{x}{x} \\
\pm\end{array}$ & $\underset{x}{x}$ & $\begin{array}{l}u_{x} \\
x\end{array}$ \\
\hline 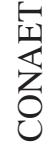 & $\begin{array}{l}u \\
x \\
x \\
y\end{array}$ & 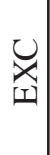 & 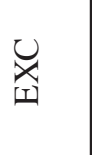 & & 峁 & $\underset{I}{x}$ & $\begin{array}{l}u \\
\underset{I}{x} \\
\end{array}$ & 岂 & 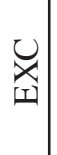 & 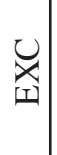 & $\stackrel{n}{\Sigma}$ & Z & $\begin{array}{l}\underset{x}{x} \\
\text { | }\end{array}$ & 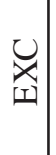 & 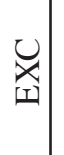 & 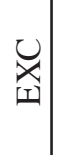 & 颃 & $\sum_{\Sigma}^{\emptyset}$ & $\sum_{\Sigma}^{\emptyset}$ & $\begin{array}{l}\bigcup \\
\underset{I}{x} \\
0\end{array}$ & $\begin{array}{l}\underset{x}{x} \\
\text { a }\end{array}$ & 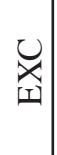 & $\underset{x}{凶}$ & 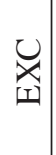 \\
\hline $\begin{array}{l}\sqrt{2} \\
\text { [II }\end{array}$ & 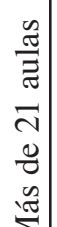 & $\begin{array}{l}\frac{\tilde{z}}{\tilde{J}} \\
\vec{\Xi} \\
\vec{\lambda} \\
0 \\
\tilde{v}\end{array}$ & 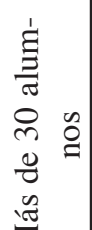 & 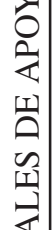 & 80 & @̊ & $\begin{array}{l}80 \\
8 \\
0\end{array}$ & $\begin{array}{l}\stackrel{0}{0} \\
\dot{q}\end{array}$ & $\begin{array}{l}0 \\
8 \\
0 \\
0\end{array}$ & $\begin{array}{l}0 \\
8 \\
0\end{array}$ & $\begin{array}{l}0 \\
6 \\
6\end{array}$ & 岂 & 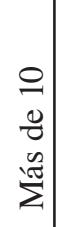 & $\begin{array}{l}a \\
\tilde{a} \\
\tilde{0} \\
\tilde{a}\end{array}$ & $\begin{array}{l}a \\
\pi \\
\infty \\
0 \\
0\end{array}$ & @̊ & $\begin{array}{l}\stackrel{0}{0} \\
0\end{array}$ & $\begin{array}{l}\text { :̊ } \\
\text { aे }\end{array}$ & $\begin{array}{l}0 \\
\infty \\
\infty\end{array}$ & $\begin{array}{l}\stackrel{0}{2} \\
\text { an }\end{array}$ & ठ̊ & $\begin{array}{l}\stackrel{0}{ } \\
\infty\end{array}$ & ஜ̊ & \begin{tabular}{l}
$\stackrel{2}{2}$ \\
\multirow{2}{*}{}
\end{tabular} \\
\hline
\end{tabular}

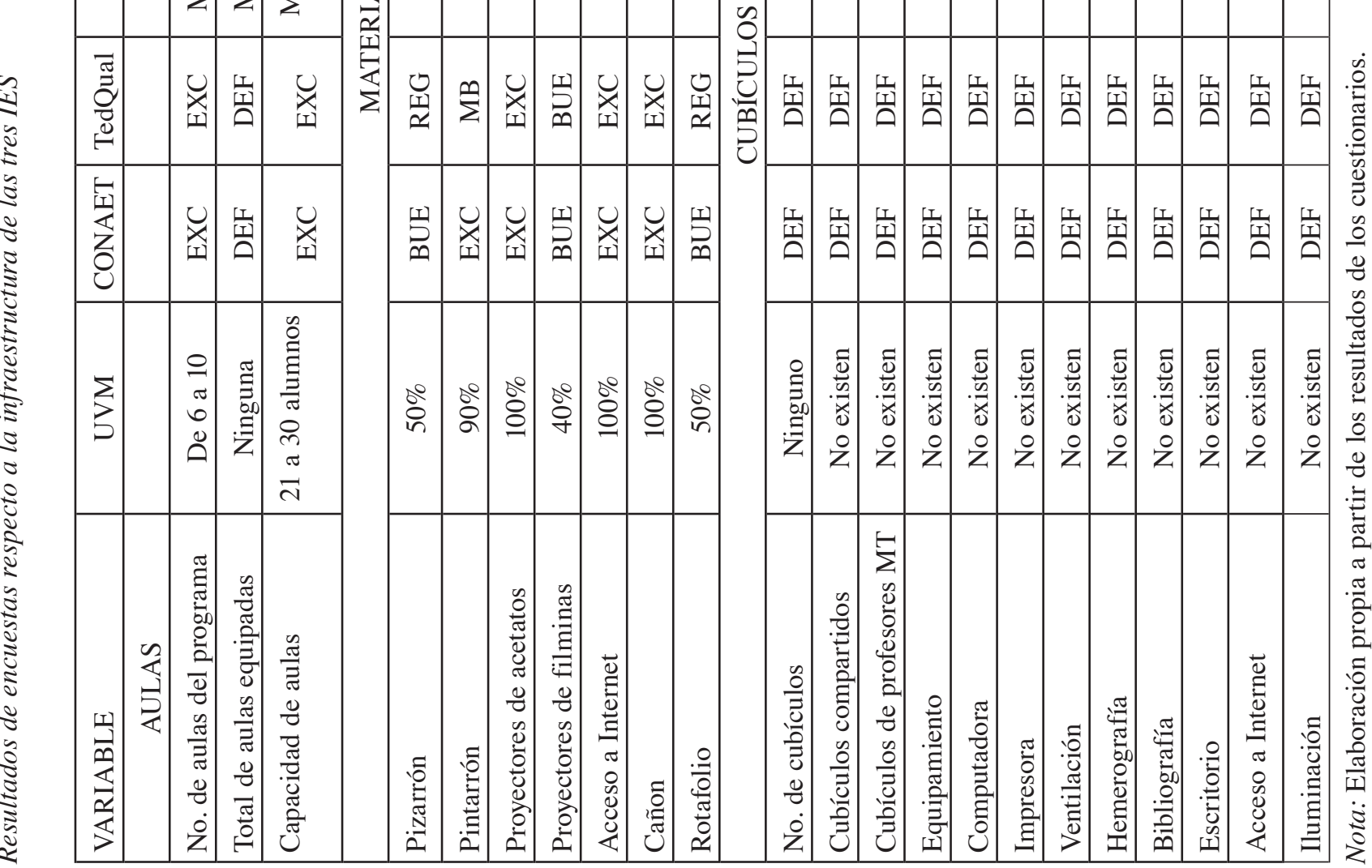


Por su parte, la infraestructura (tabla 3) tiene como principales debilidades un servicio deficiente para la UVM, en cuanto al mantenimiento a las computadoras y la falta de software legal y regular para la EBC, ya que no cuenta con salas de esparcimiento y descanso para docentes. En lo referente a salas de audiovisuales, las tres instituciones cuentan con ellas; sin embargo, en el caso de la UVM no se encuentran acondicionadas y equipadas con lo mínimo requerido por los organismos certificadores y el uso que se les da -tanto en la UVM como en la EBC- es diverso. En lo referente a centros de información, estos son compartidos por las demás carreras ofrecidas por la UVM y EBC, así como no se cuenta con un acervo adecuado en el área de turismo ni tampoco se encuentra actualizado. Por su parte, la EST-IPN no presenta deficiencias respecto al material con el que se cuenta, además de ser una biblioteca depositaria de la OMT, lo que permite ampliar de forma constante sus acervos y, por ende, la actualización de estos.

En lo que respecta a los laboratorios de agencias de viajes y hotelería (Tabla 4), se puede observar que tanto la UVM como la EBC carecen de laboratorios de este tipo, lo que limita el aprendizaje de los jóvenes en cuanto a los procesos formativos; en tanto la EST sí posee dichos laboratorios. Sin embargo, la EBC es una institución privada que cuenta con un convenio con el Hotel City Express, el cual alberga a sus estudiantes en sus instalaciones para sus prácticas profesionales. La EST y UVM presenta una deficiencia ya que no tienen este equipamiento.

En lo que concierne al personal docente (tabla 5), se aprecia que la UVM cuenta con personal femenino en su mayoría, en tanto que la EST y la EBC cuentan con mayoría de profesores del sexo masculino. La asiduidad a clases por parte del personal académico va del 51 al 75\%, correspondientemente en las tres instituciones. Asimismo, tanto la UVM como la EBC no poseen profesores de tiempo completo; por el contrario, en la EST labora, en esa condición, un $60 \%$. En lo referente al nivel académico del personal académico, se aprecia que tanto en la UVM como en la EBC, el nivel máximo de estudios es de licenciatura y en algunos casos con especialidades; mientras que la EST muestra estudios que van desde licenciatura hasta doctorado.

De acuerdo con la tabla 6, la EBC expone los más bajos requerimientos en cuanto a antigüedad de docentes, la UVM presenta debilidades en el ámbito de horas dentro del programa, y la EST muestra una debilidad en cuanto a los años de servicio en el programa de turismo. En el rubro de las horas dedicadas a la docencia, tanto la UVM como la EBC evidencian un déficit en el tiempo dedicado a la actividad docente y en lo referente a la pertenencia en organismos de investigación, ya que, al no contar con líneas de investigación, tampoco se encuentran dentro de este tipo de organizaciones. Mientras que para el caso de la EST, existen profesores con investigaciones, pero no pertenecen al SNI y al AMIT.

Como consecuencia de una carencia de líneas de investigación, la UVM y EBC carecen de productos académicos, asistencia a congresos como asistentes y menos aún como ponentes, lo cual también conlleva a no dedicarse a la investigación, con la consabida limitación en su acción docente, pues tan solo se queda con conocimientos empíricos. Por su parte, la EST muestra una deficiencia en cuanto a publicación de resultados en revistas nacionales o internacionales, lo que acarrea la no divulgación de los resultados de dichas investigaciones (Tabla 7).

En lo concerniente a la supraestructura de las instituciones (Tabla 8), se tiene que las tres evidencian deficiencias en cuanto al conocimiento de la misión y visión. Respecto al modelo educativo, la UVM muestra las mayores carencias a nivel de administrativos y docentes y, en menos medida, la EBC. Las similitudes en cuanto al conocimiento del modelo educativo y procesos de evaluación es deficiente en ambas instituciones privadas. 


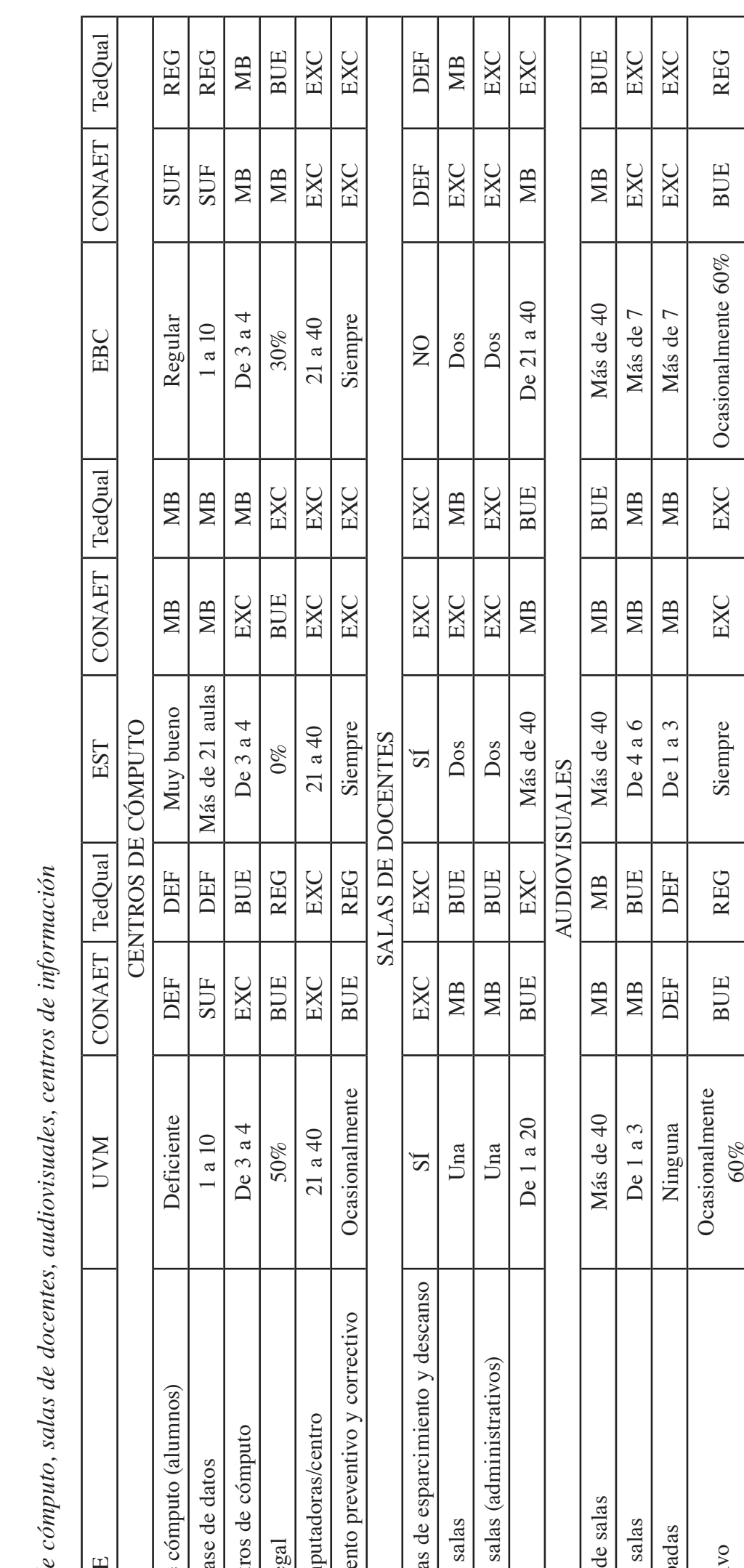

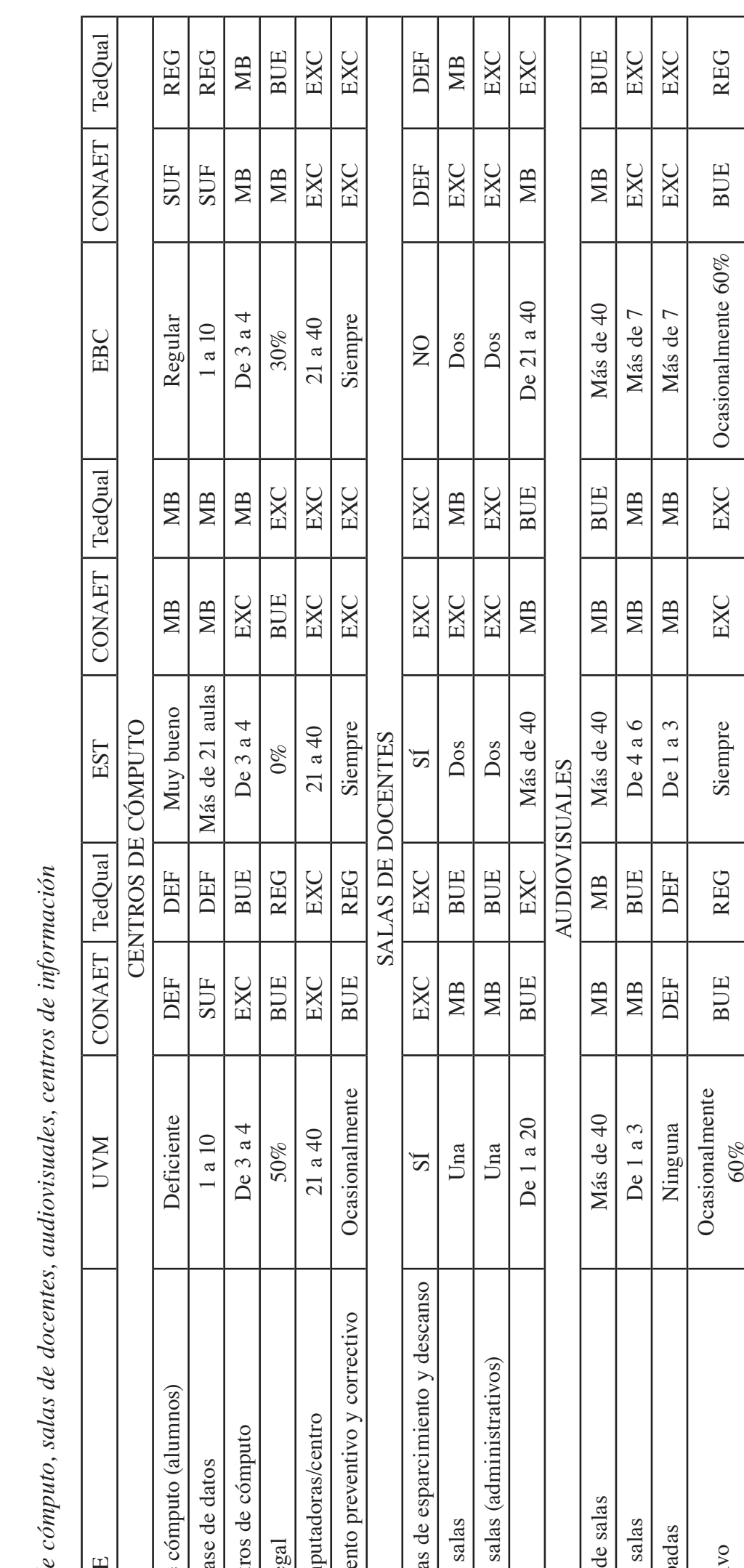

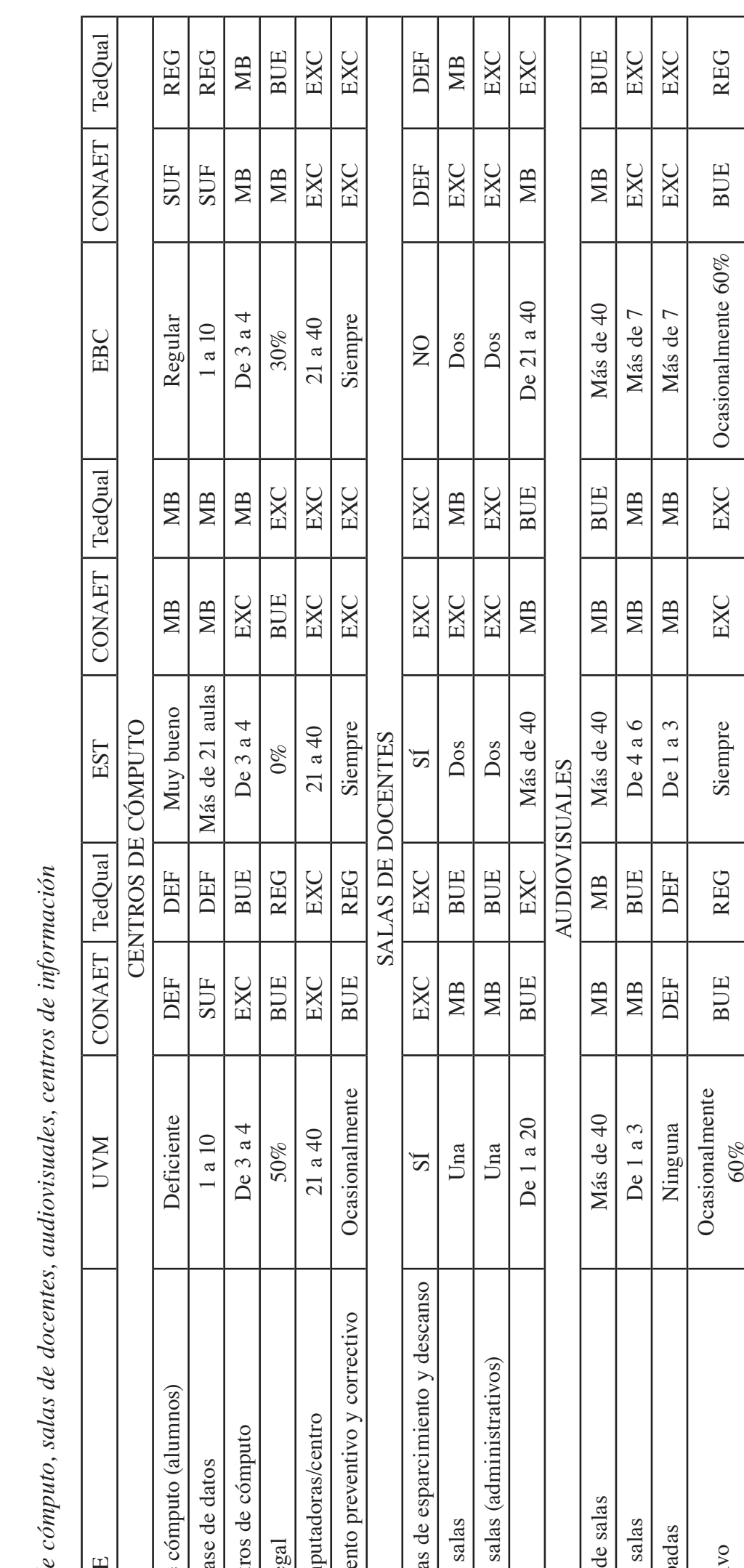

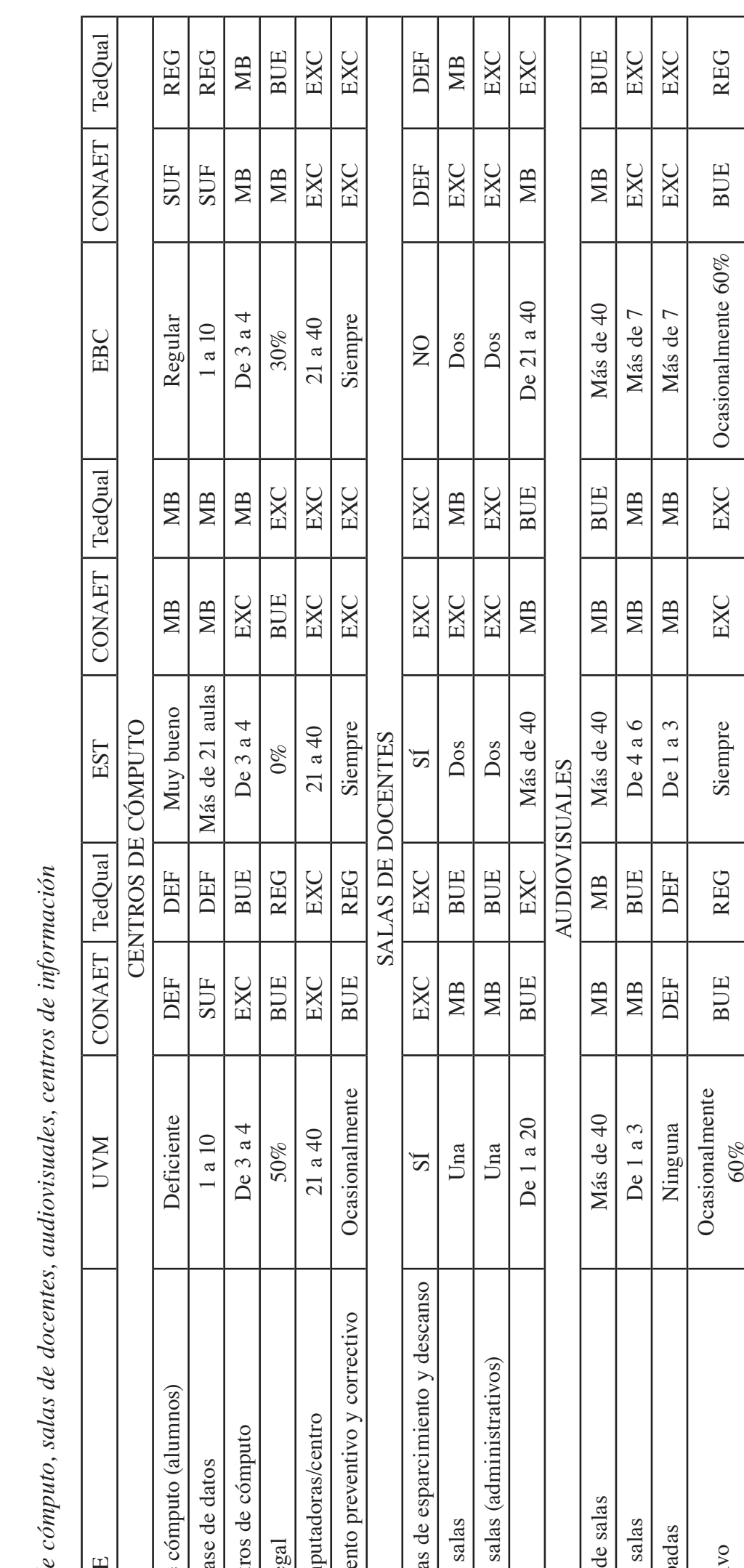

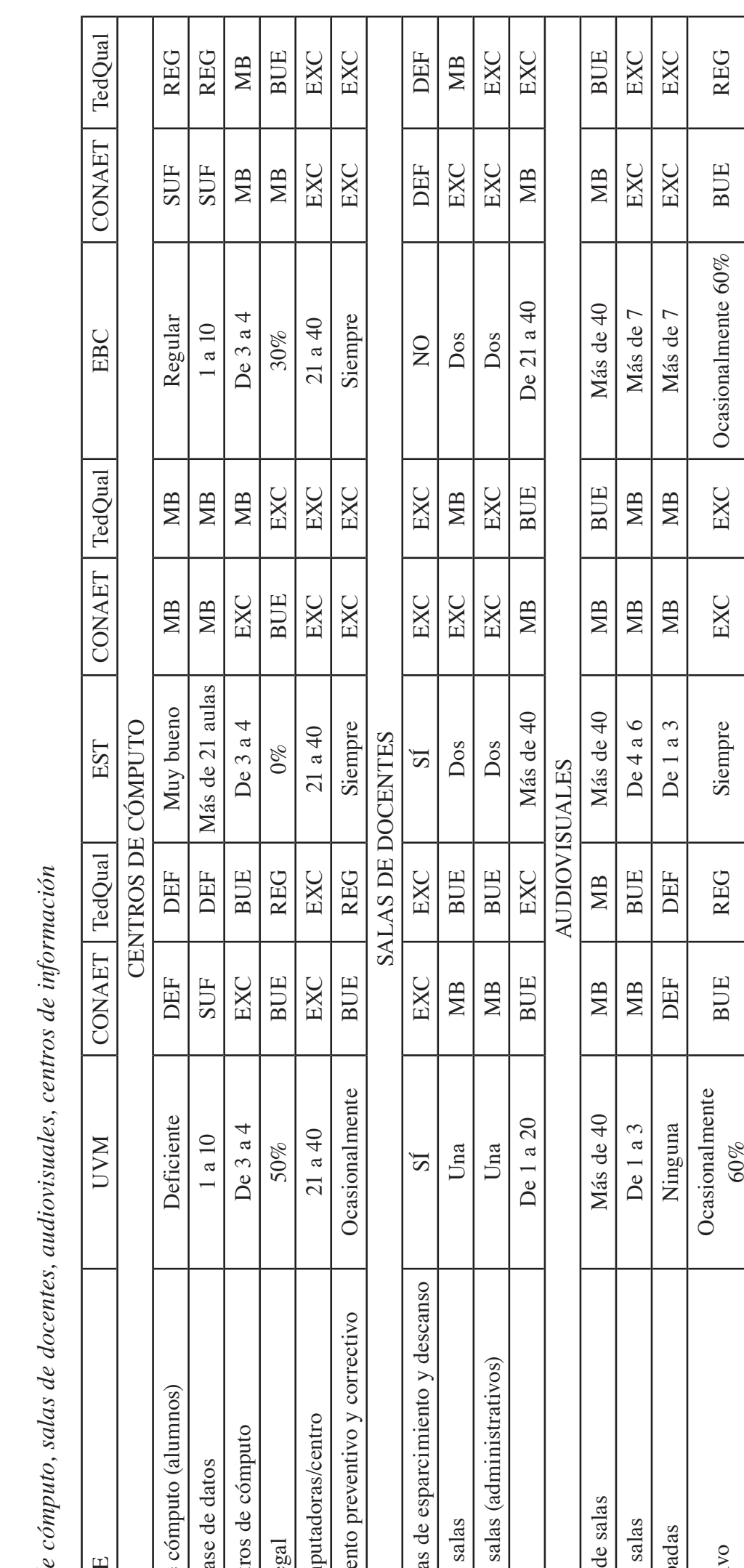

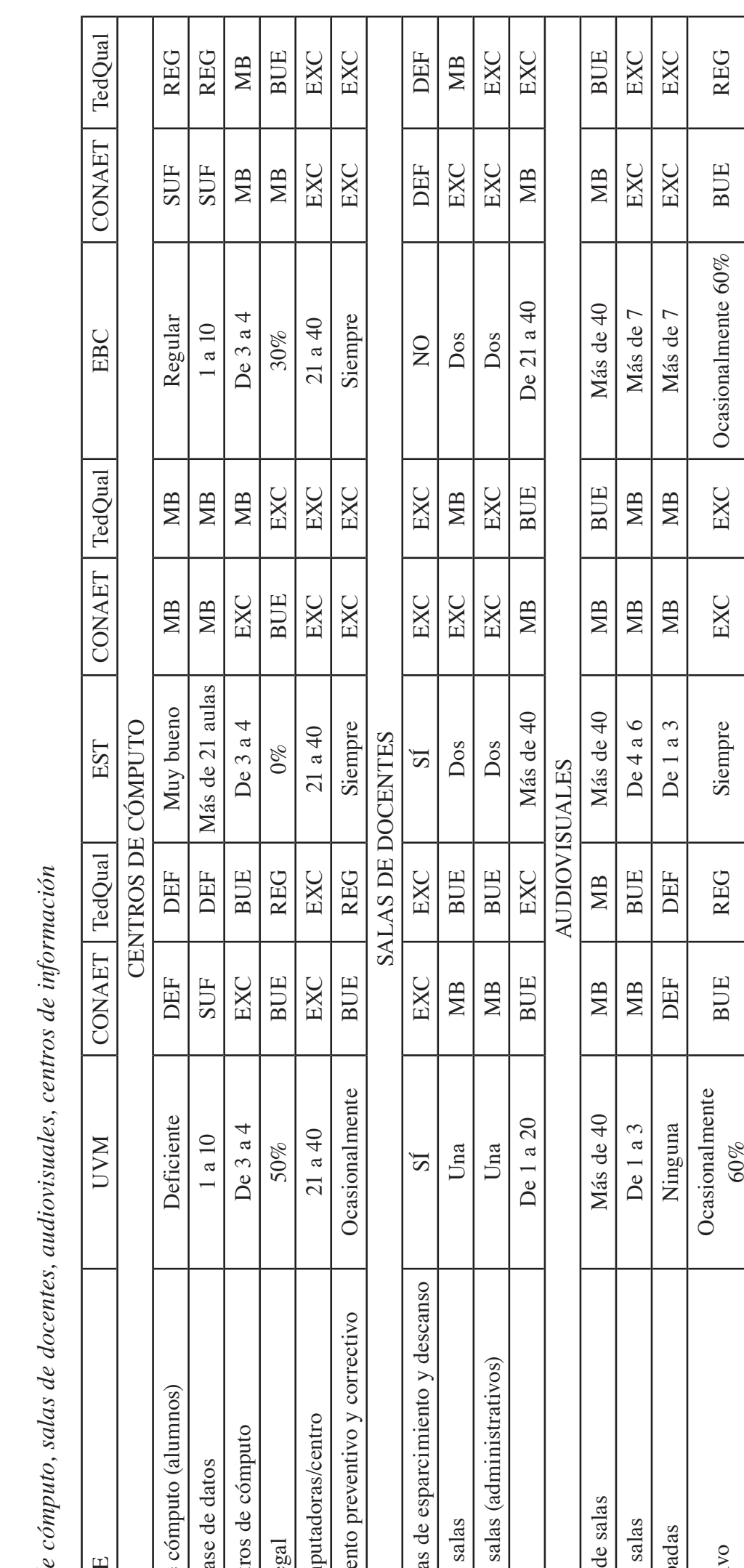

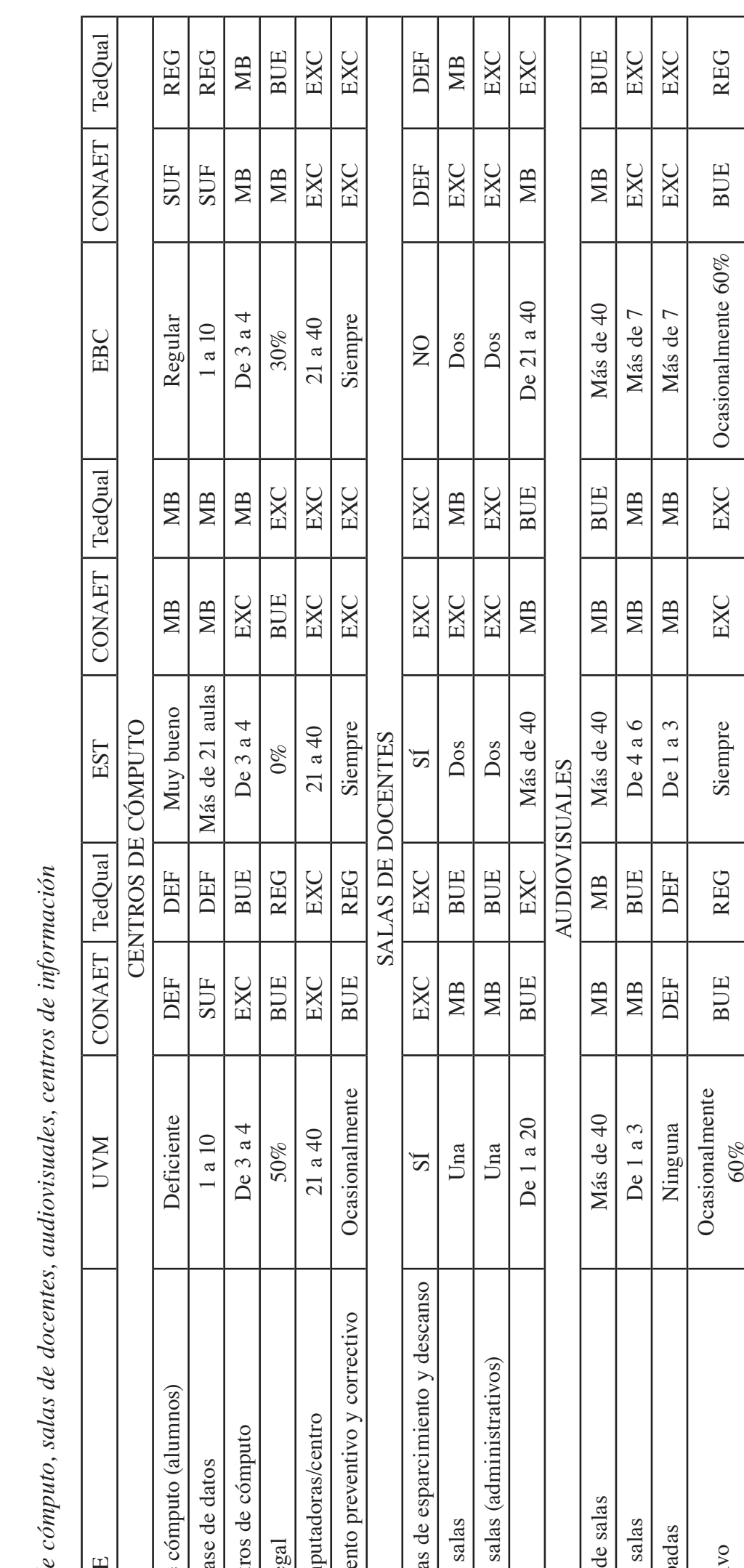

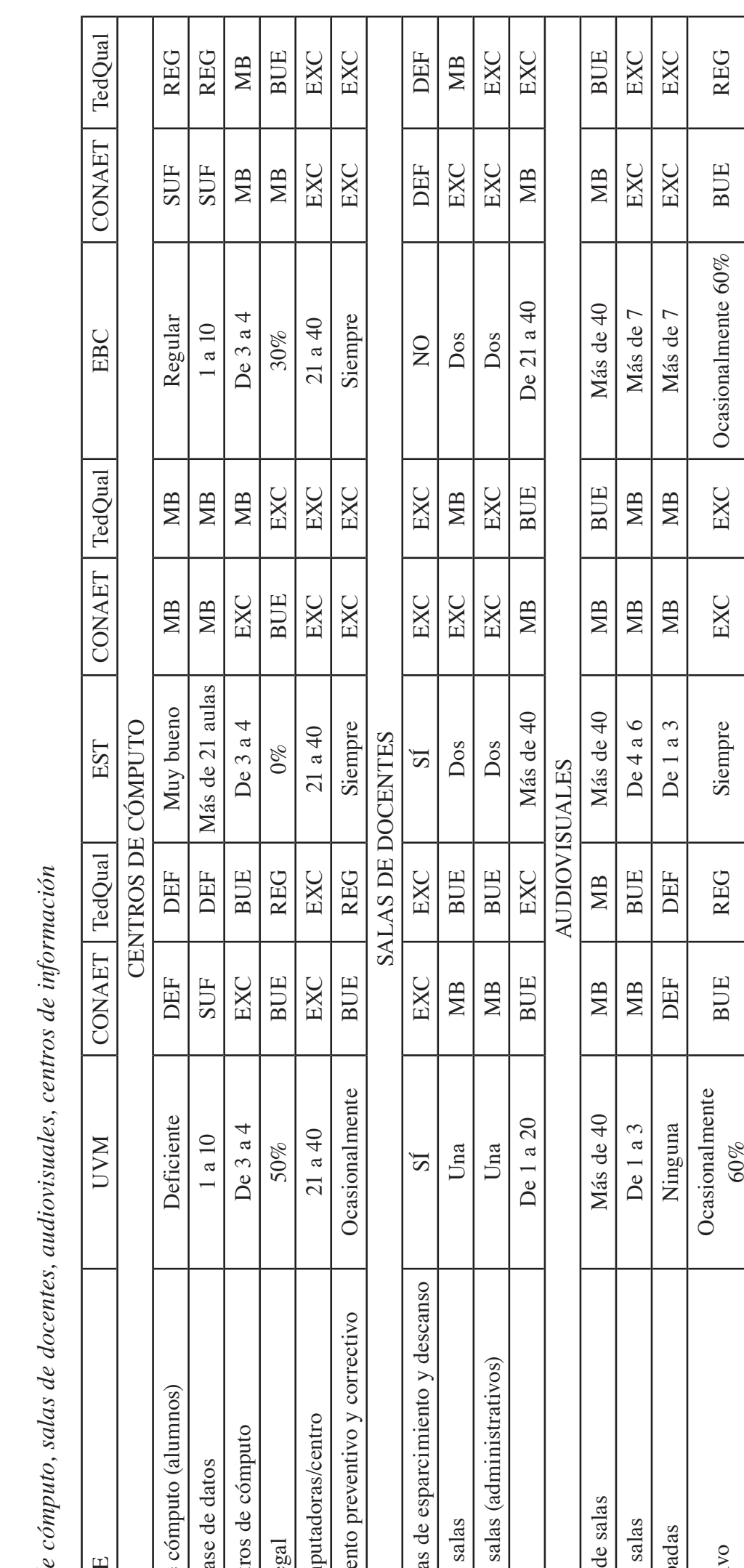

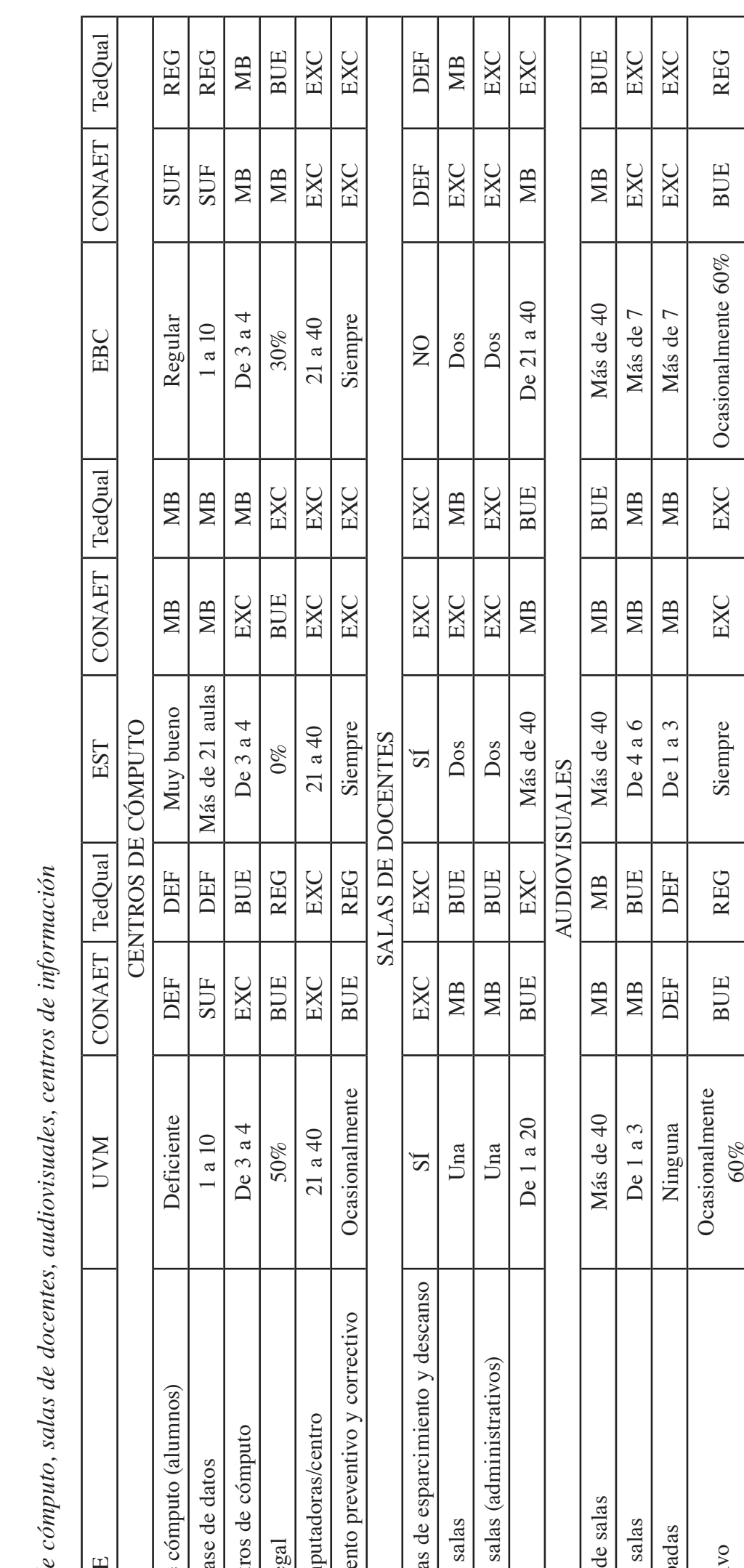

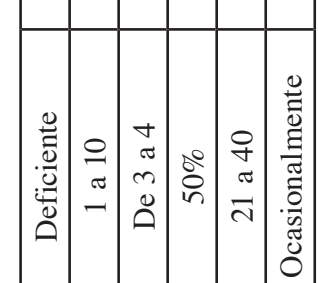

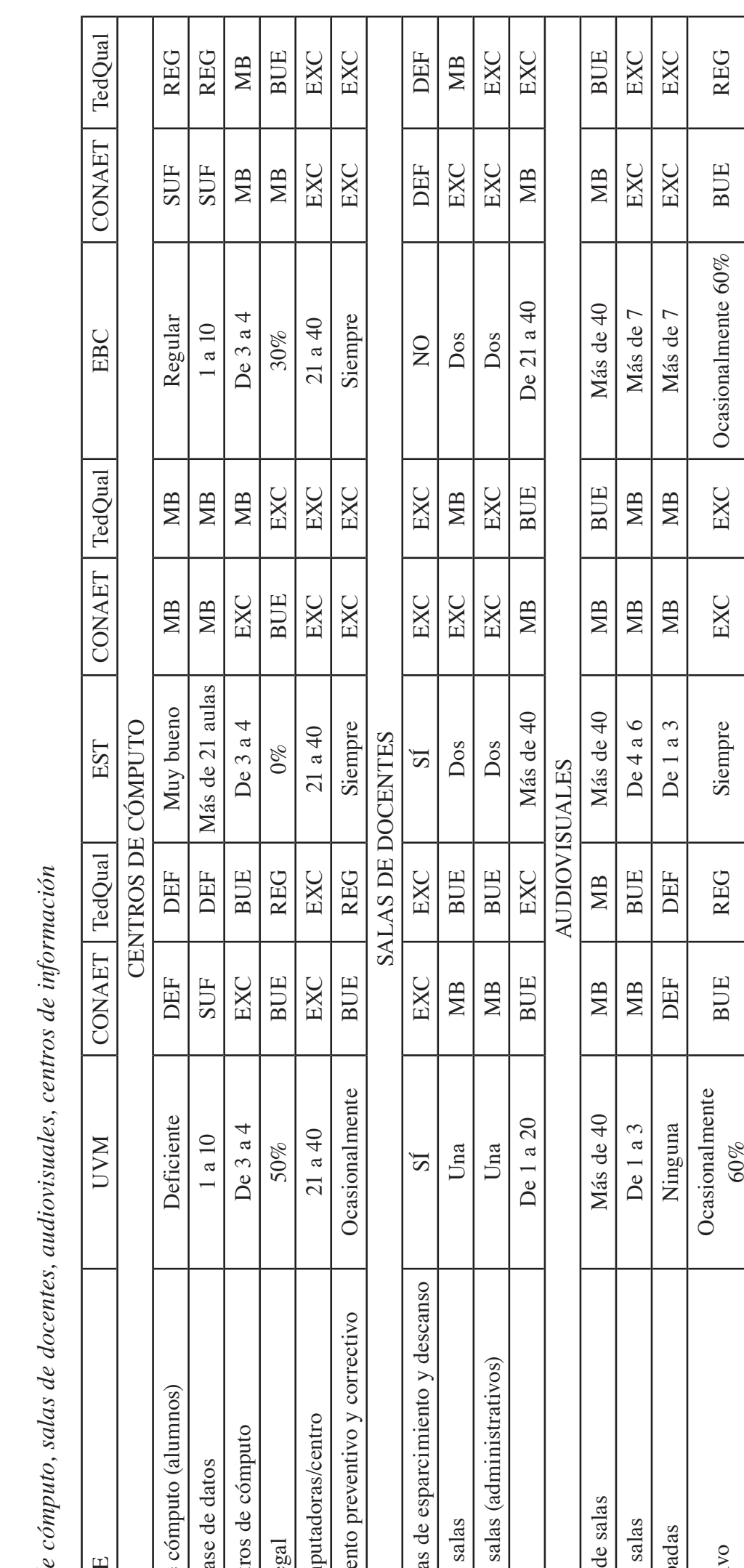

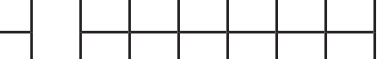

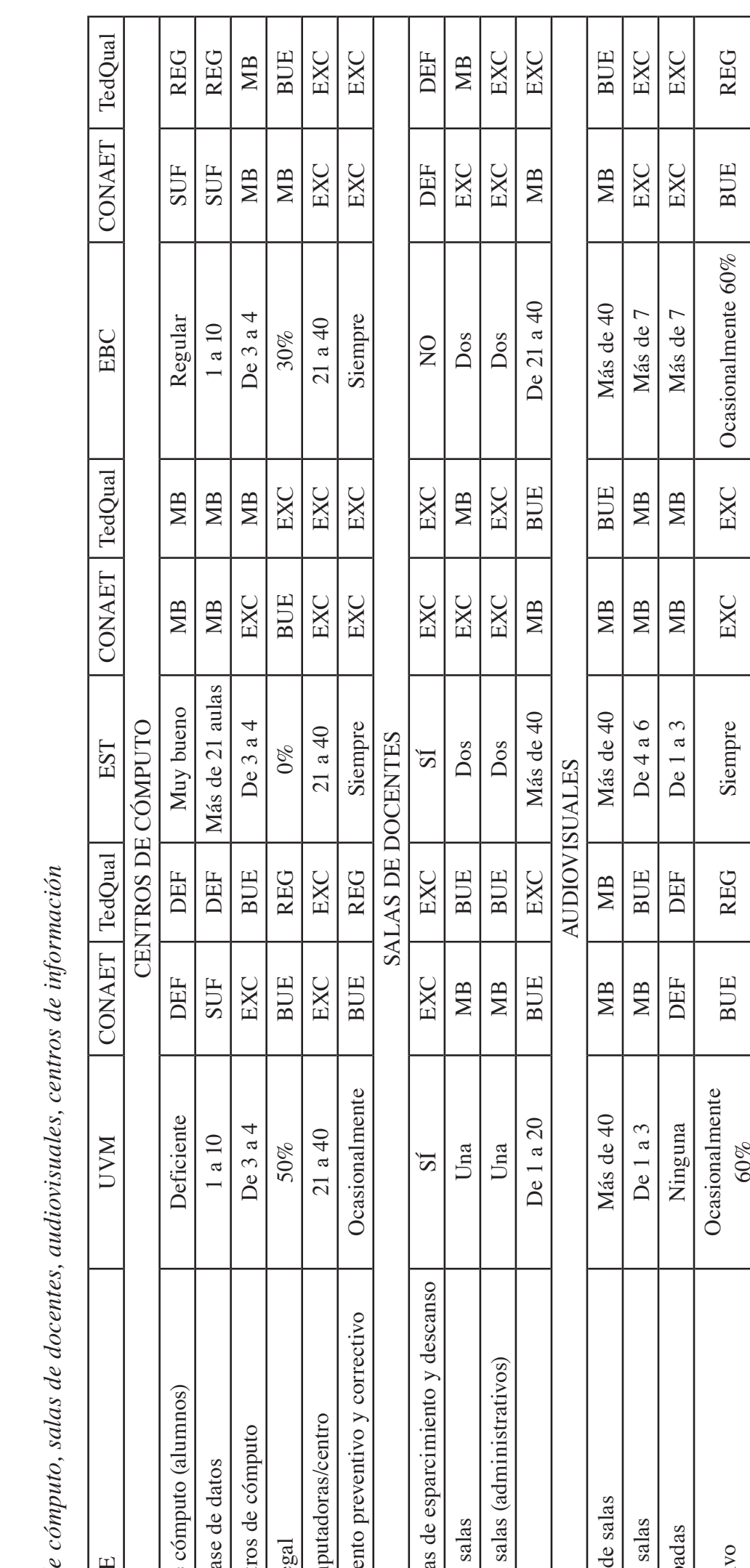

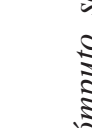

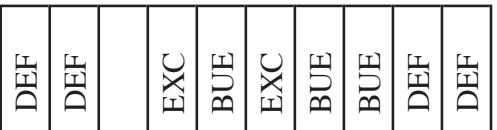

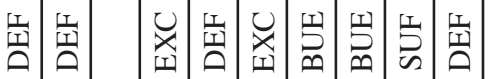

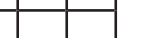

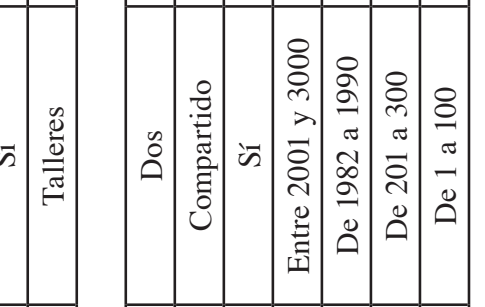

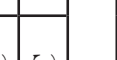

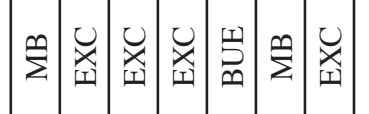

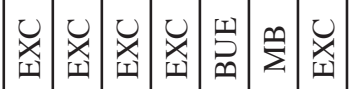




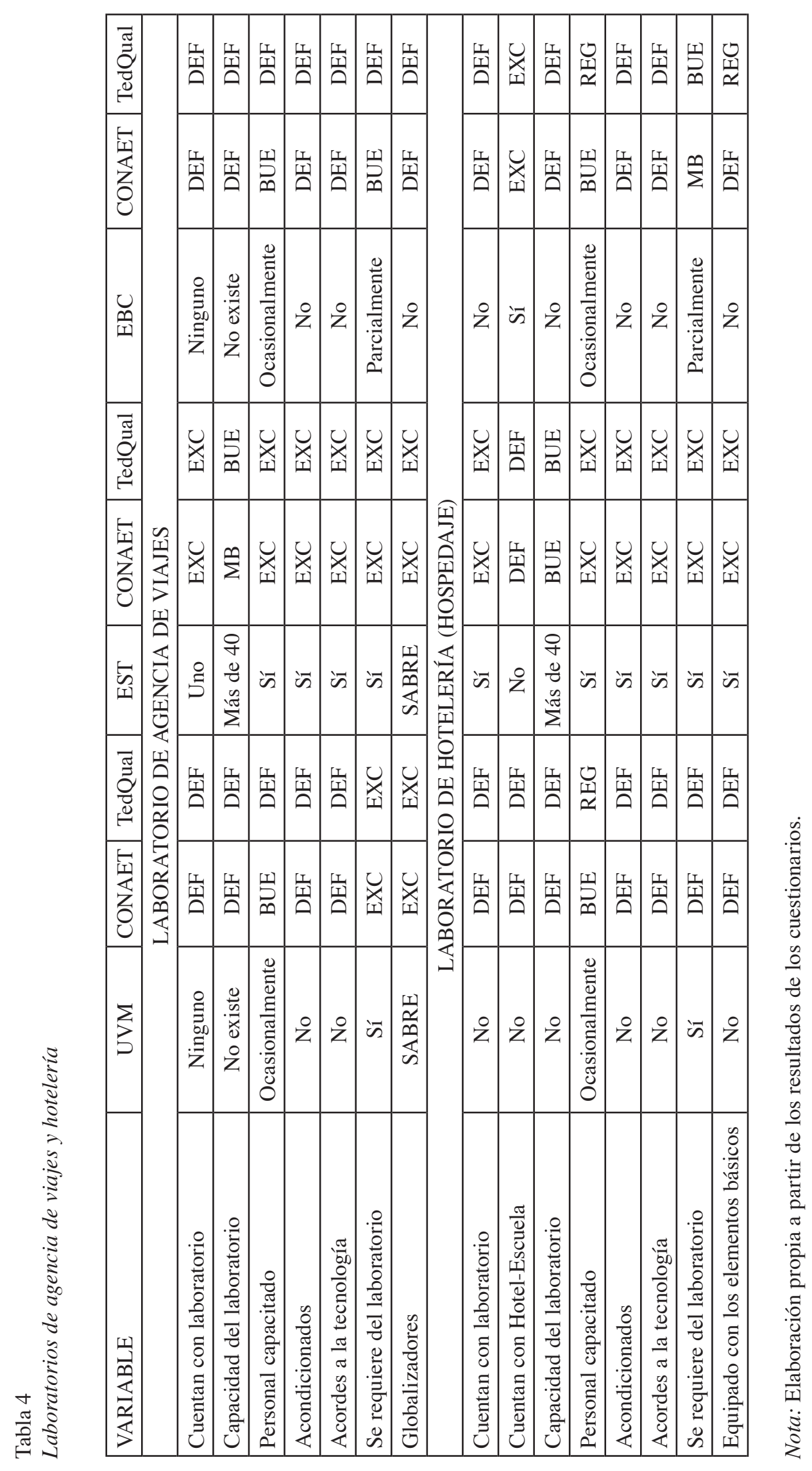




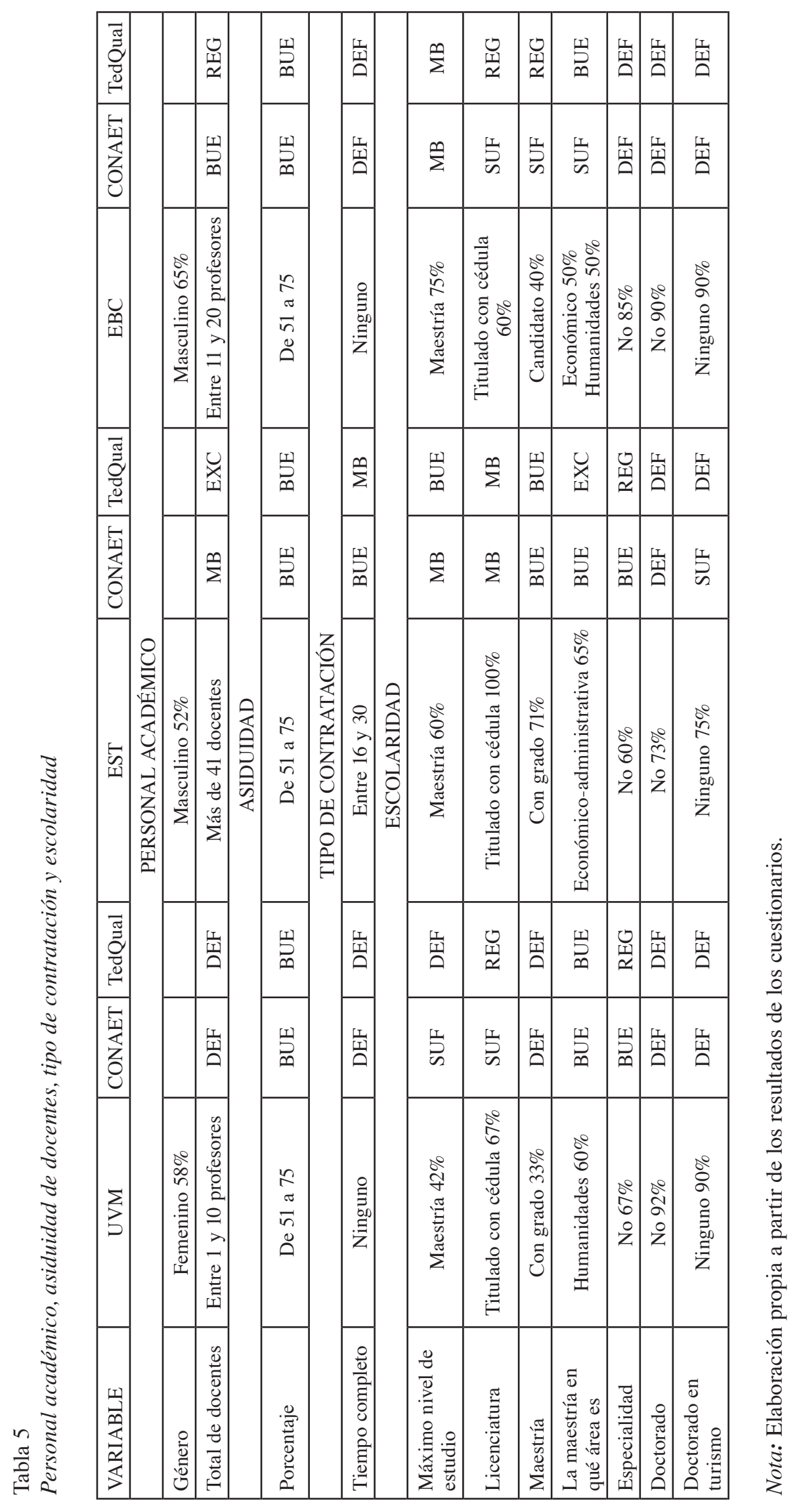




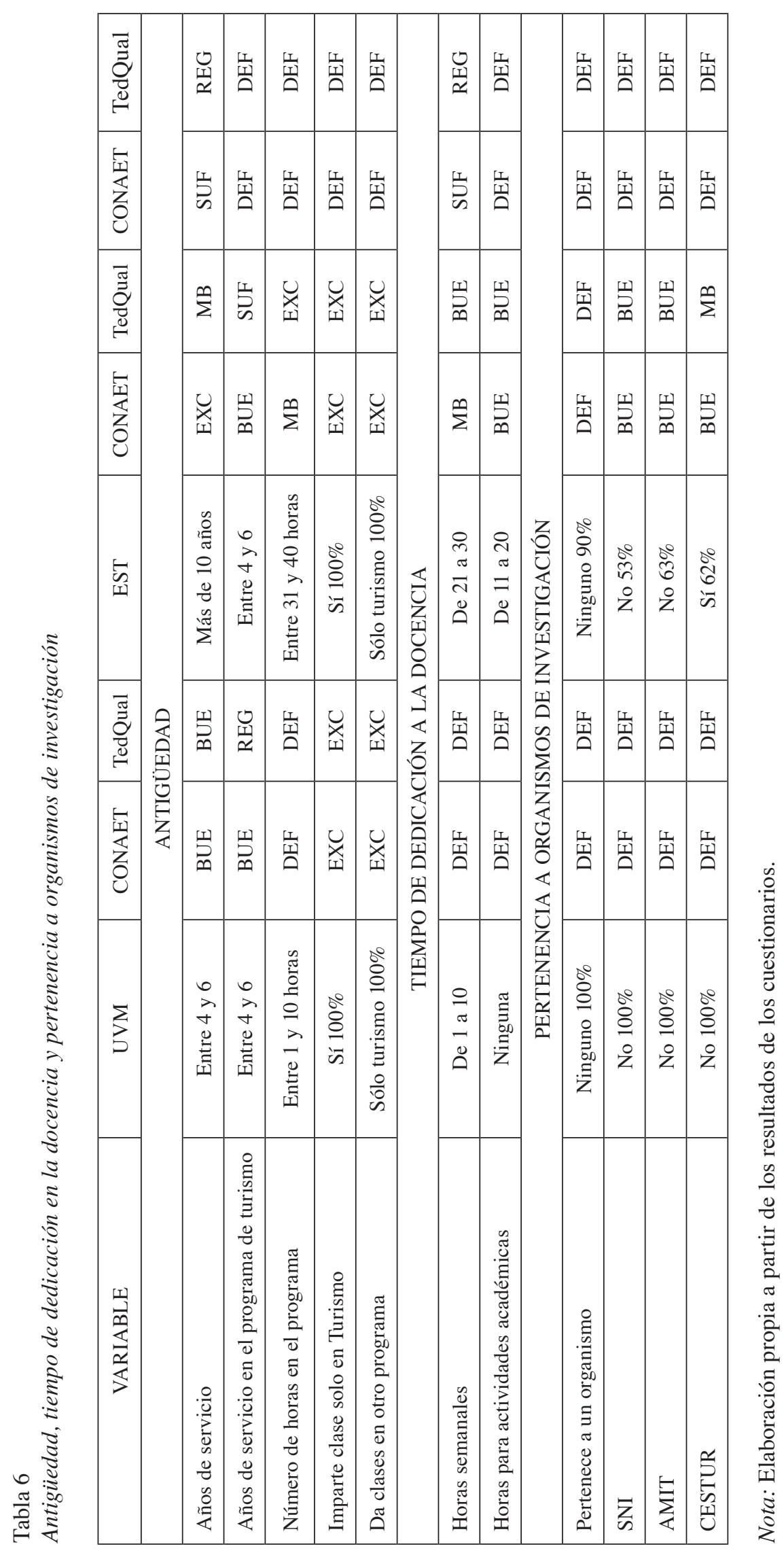




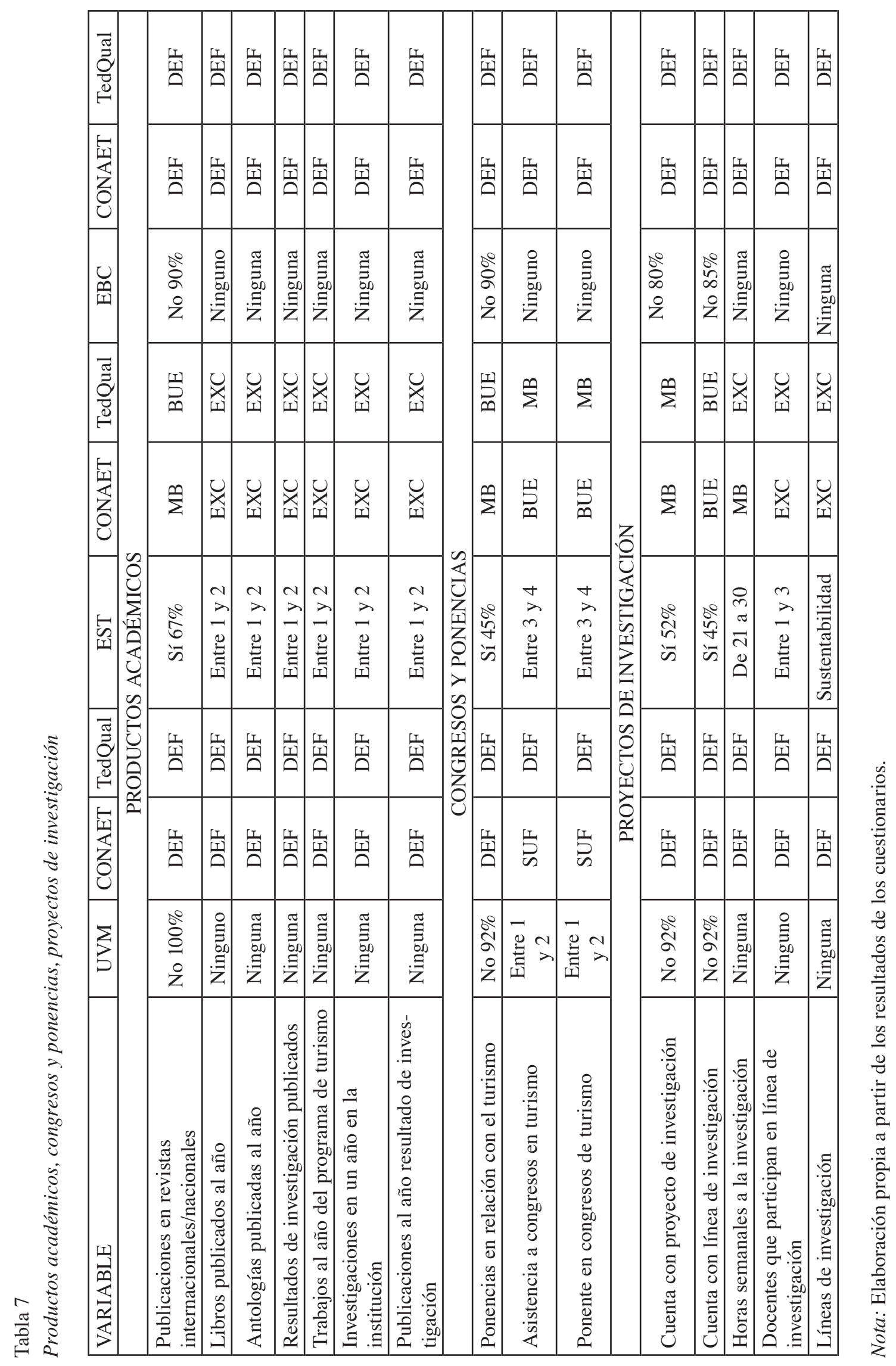




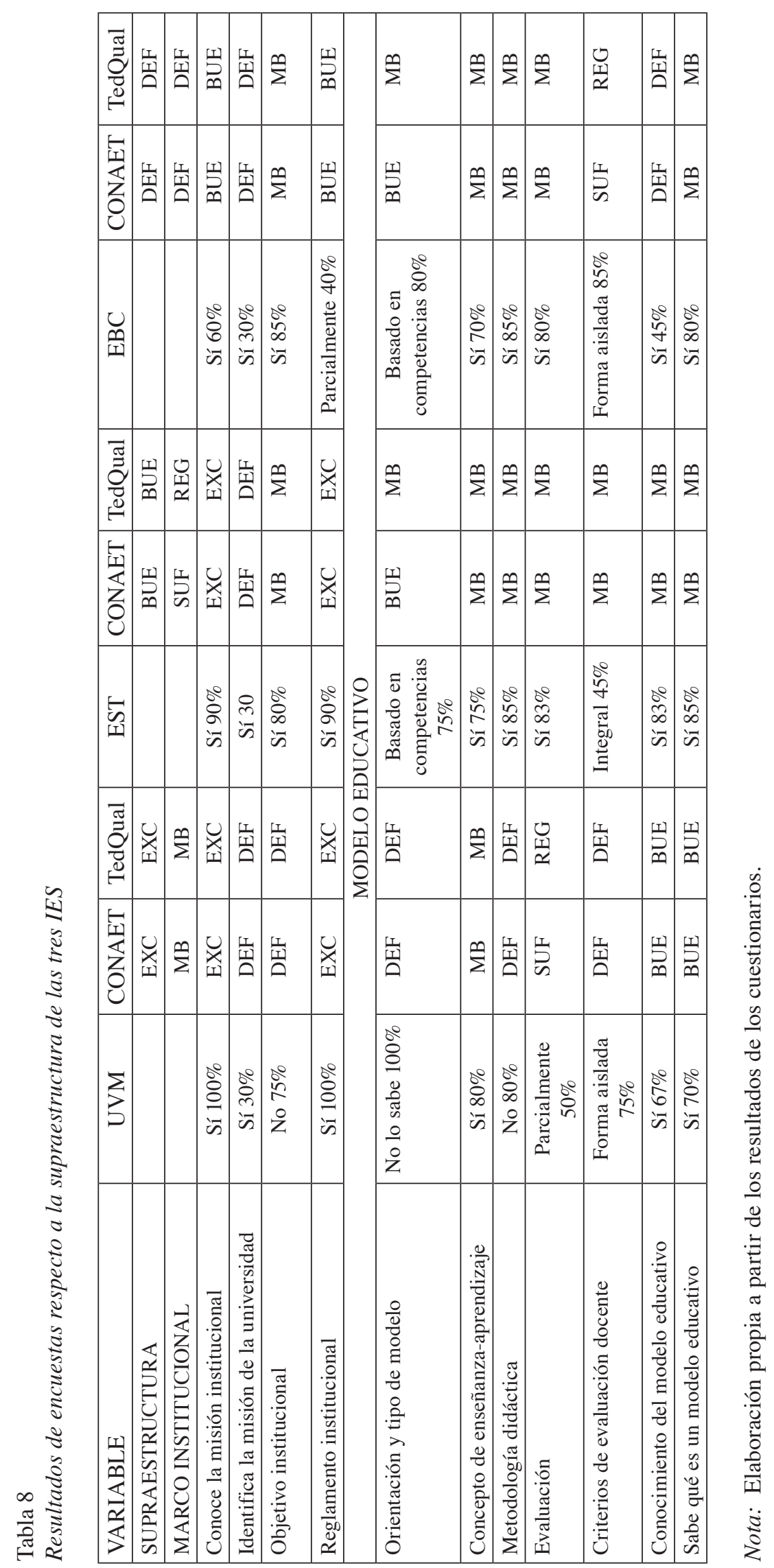


De las guías de observación

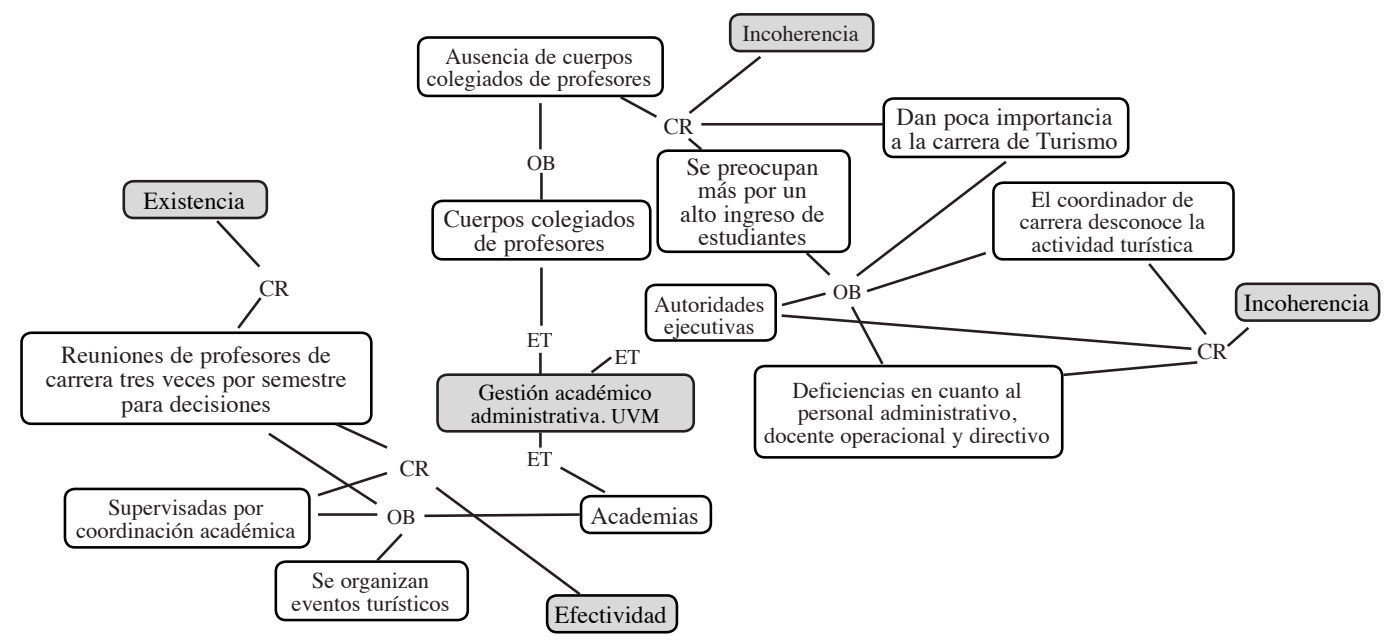

Figura 2. Gestión académico administrativa de la UVM.

Nota: Elaboración propia a partir de los resultados de guías de observación.

Para el caso de la gestión académico administrativa (figura 2), se hace necesario que existan órganos que regulen ambos procesos, por lo que los cuerpos colegiados son necesarios para direccionar las acciones de la educación al interior de la institución y, por ende, la toma de decisiones. Sin ello difícilmente se conducirá hacia el logro de los objetivos organizacionales.

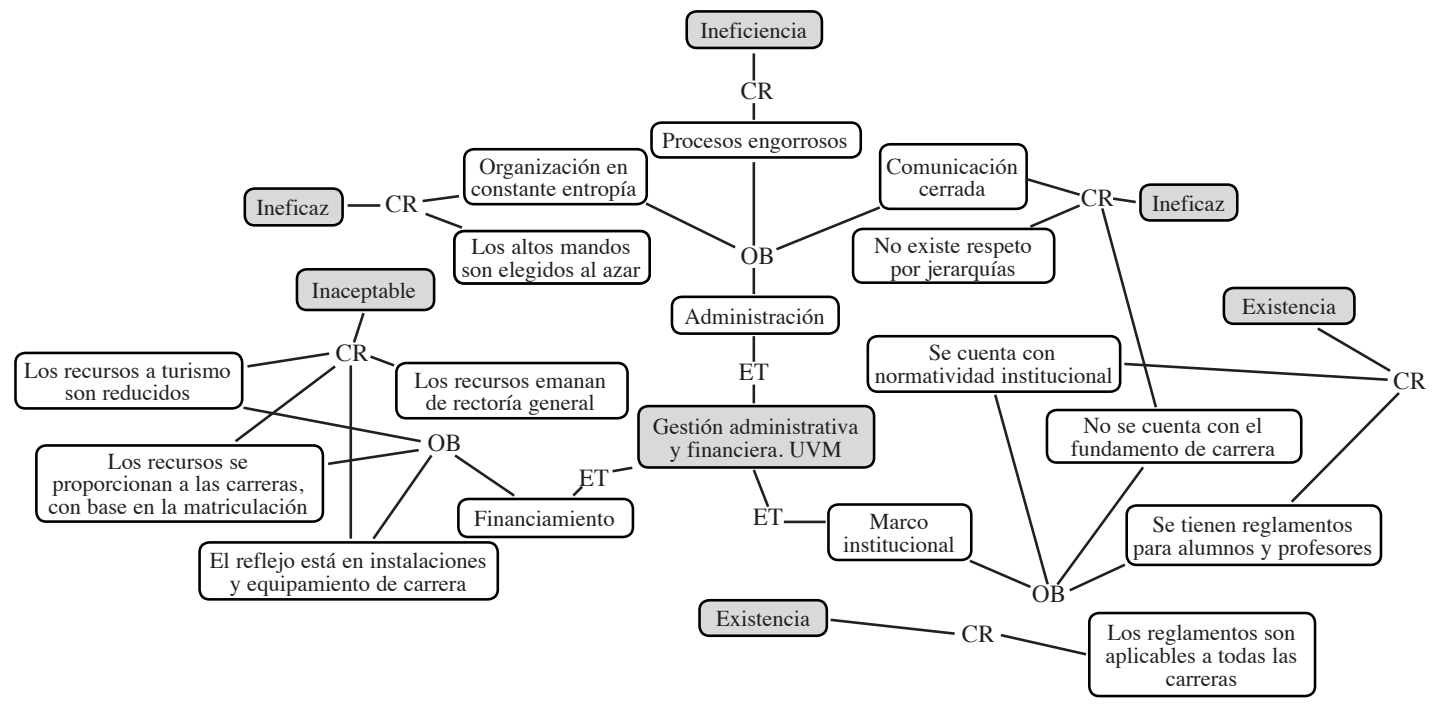

Figura 3. Gestión administrativa y financiera de la UVM.

Nota: Elaboración propia a partir de resultados de guías de observación.

Para el caso de la gestión administrativa y financiera (figura 3), no existe una selección acorde con las necesidades de la institución, pues la gran mayoría de los directivos no poseen experiencia en este rubro o, simplemente, son removidos de sus cargos a otros campus, por lo que se percibe que cumplen con sus labores por el simple hecho de cumplir, lo que conlleva a una entropía elevada en todos los aspectos al interior de cada área de trabajo. En lo referente a los recursos, estos emanan de la rectoría general y son repartidos con base en la matrícula obtenida en un periodo específico, lo que refleja un bajo presupuesto de la carrera de turismo, por sus escuetas instalaciones y equipamiento. 


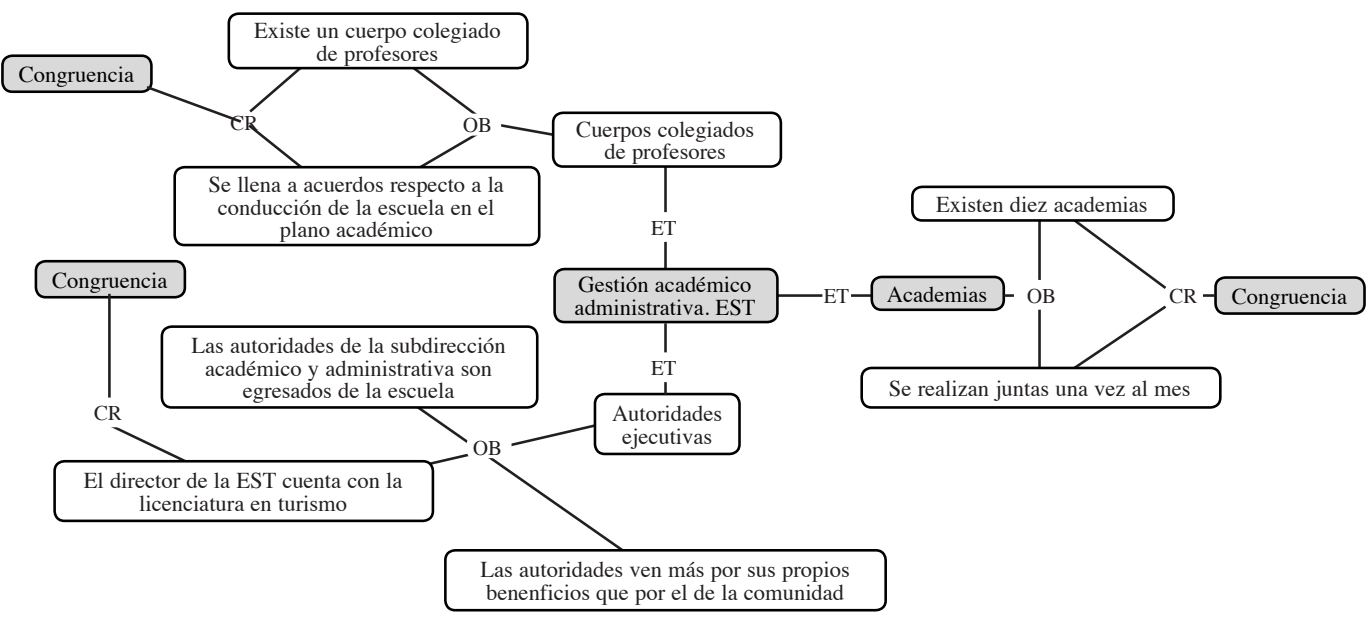

Figura 4. Gestión académico administrativa de la EST

Nota: Elaboración propia a partir de resultados de guías de observación.

Como se muestra en la figura 4, la gestión académico administrativa evidencia poseer las autoridades ejecutivas y los órganos colegiados académicos adecuados para el desarrollo del programa, todo ello sustentado en la normatividad institucional. Asimismo, estos participan en la toma de decisiones sobre los procesos de análisis y aprobación de las políticas del quehacer académico y de dirección del proceso educativo, de acuerdo con las responsabilidades que establece el marco jurídico.

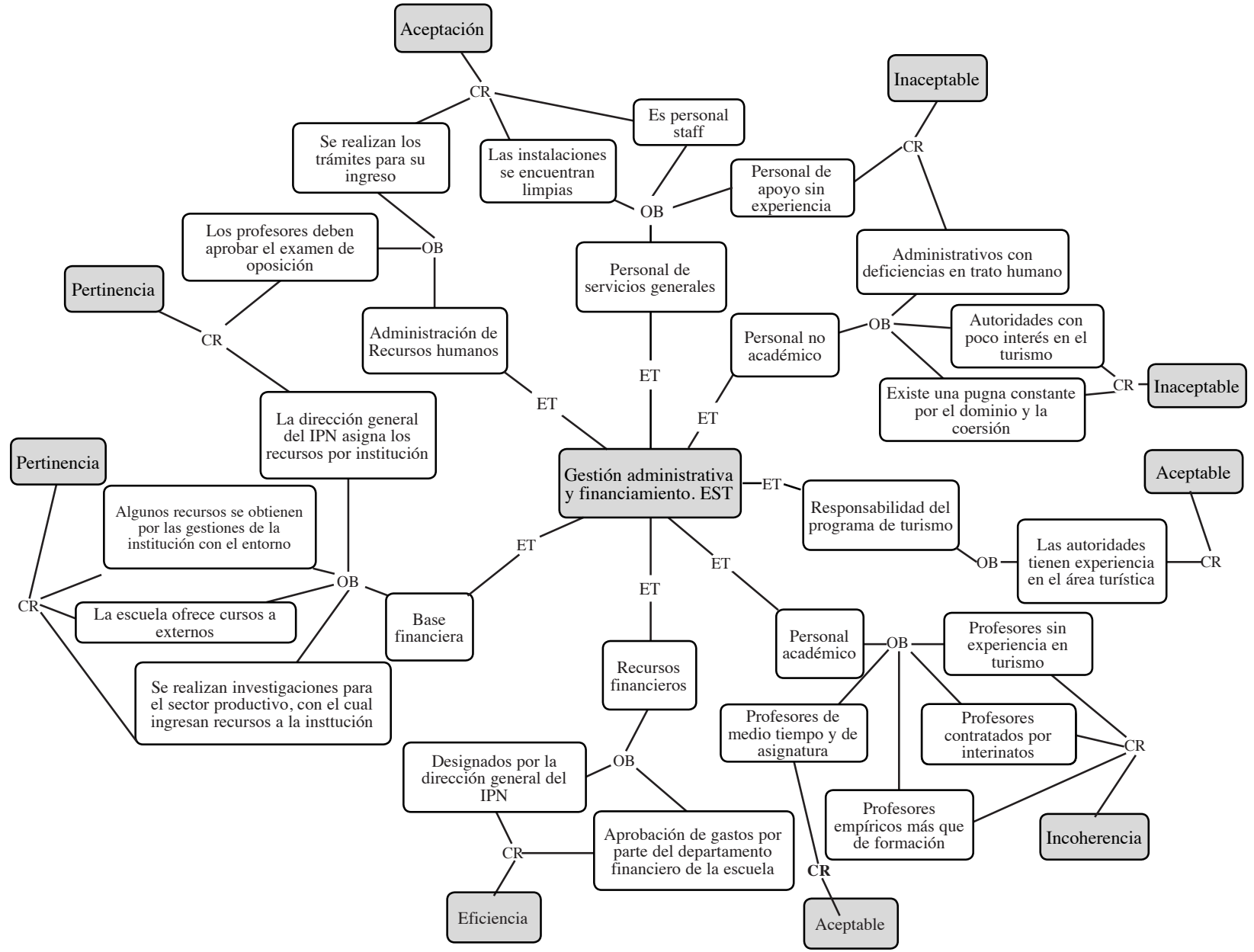

Figura 5. Gestión administrativa y financiamiento EST.

Fuente: Elaboración propia a partir de resultados de guías de observación. 
Respecto a la figura 5, en relación a la gestión administrativa y financiamiento, el ejecutivo federal asigna los recursos financieros a las IES, siguiendo el Modelo Oficial de Asignación del Subsidio Público. Este tiene criterios diversos para otorgarlos y comprende: el subsidio ordinario, que cubre el gasto corriente asignado para sostener la operación regular de las instituciones; y el subsidio extraordinario, que son los recursos destinados a mejorar y asegurar la calidad de la educación, mediante una serie de programas como cursos externos e investigaciones con el sector productivo.

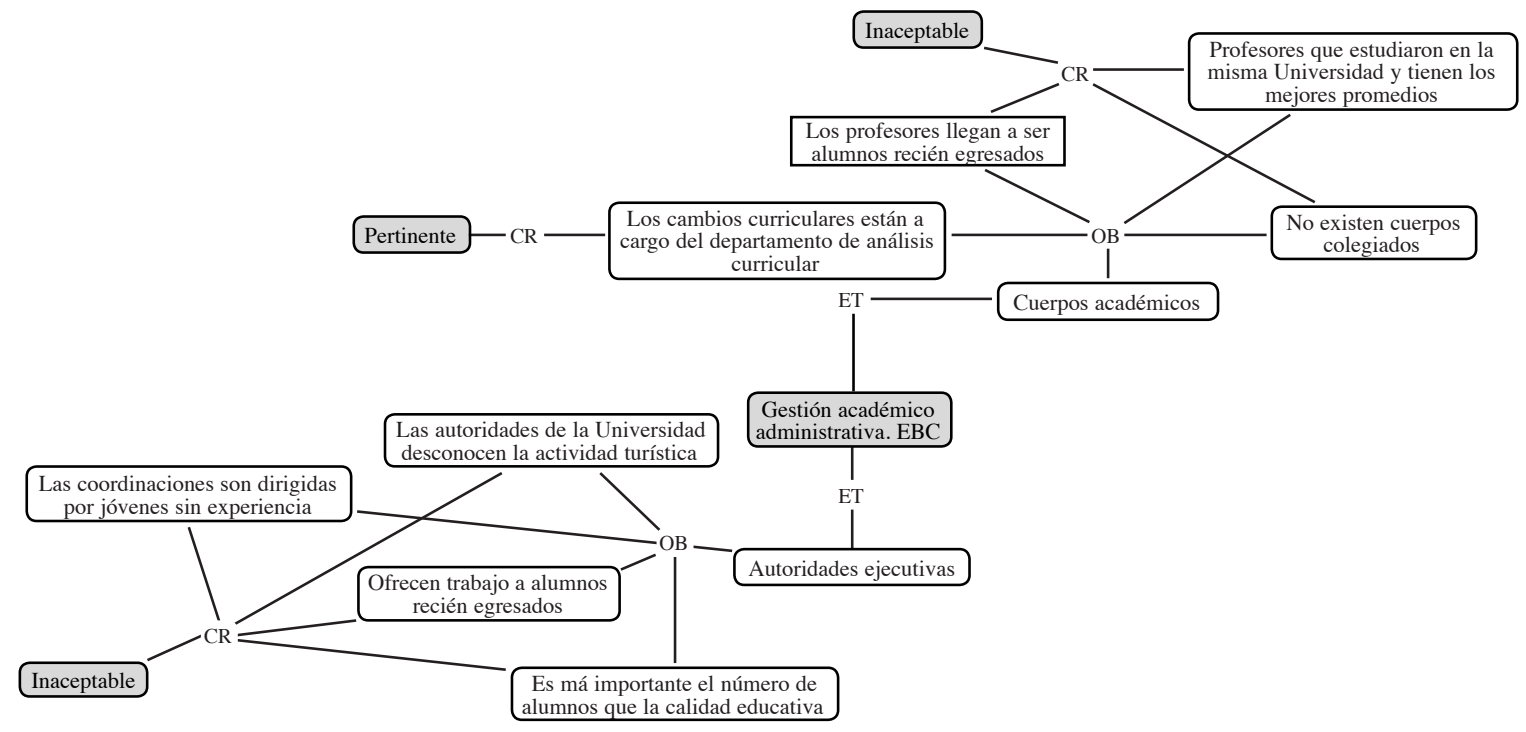

Figura 6. Gestión académico administrativa de la EBC.

Nota: Elaboración propia a partir de resultados de guías de observación.

En lo referente a la gestión académico administrativa de la institución (Figura 6), se observan debilidades en cuanto a: 1) Cuerpos académicos colegiados: no existen al interior de la institución, lo que repercute en la aplicación, objetivos, métodos y toma de decisiones. 2) Autoridades ejecutivas: desconocen el sector turístico y las coordinaciones están a cargo de jóvenes recién egresados de la institución. Indiscutiblemente se hace necesario conformar cuadros de personal altamente competitivo, comprometido con la organización educativa y, sobre todo, con capacidades inherentes a sus cargos.

La gestión administrativa y financiera de la EBC (Figura 7) presenta las siguientes debilidades: a) Los recursos asignados a la carrera de turismo y hotelería corresponden al $7 \%$ promedio anual. b) Los procesos administrativos tienen deficiencias en cuanto al incumplimiento de reglas, políticas y estatutos de la universidad. 


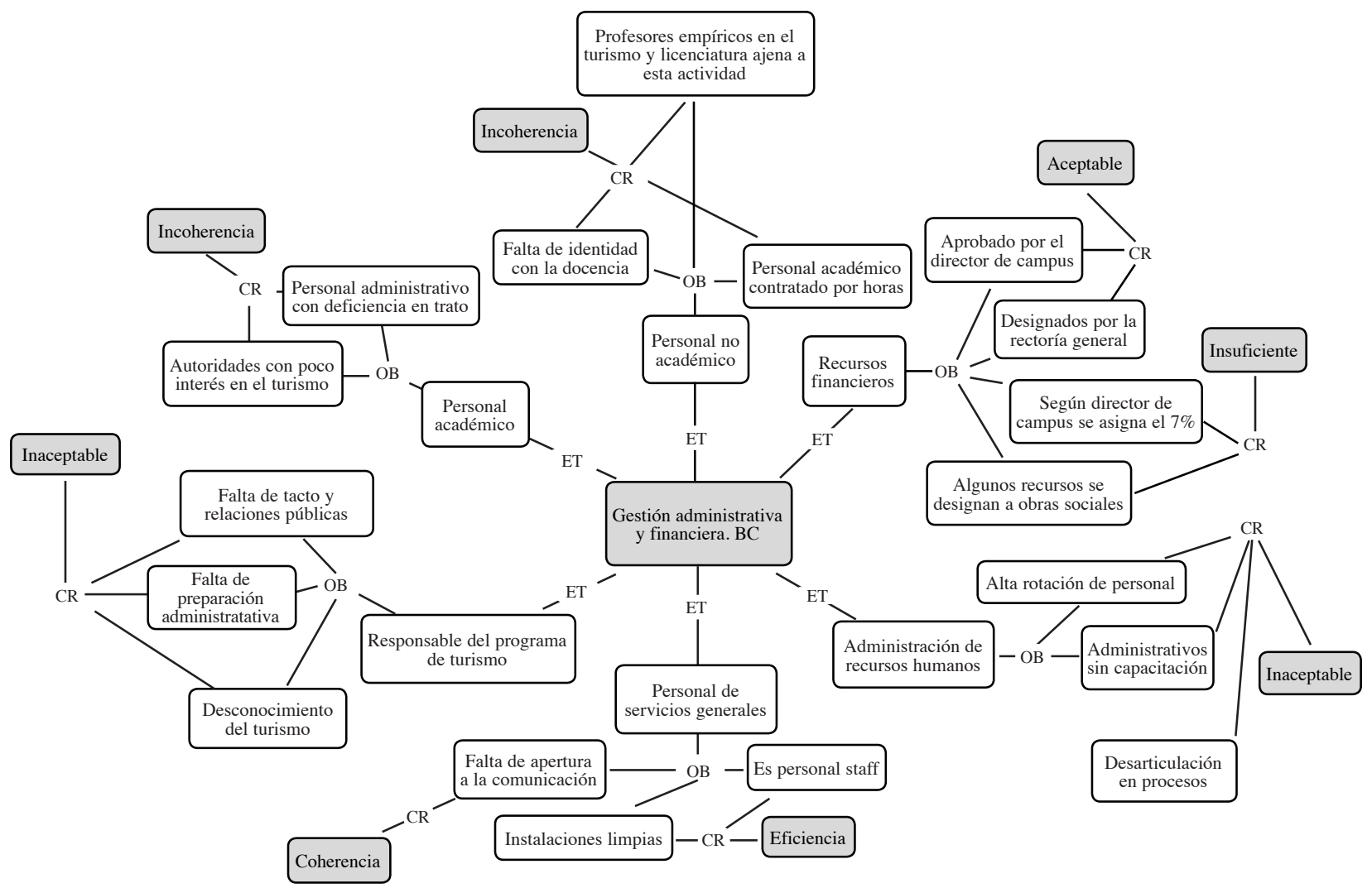

Figura 7. Gestión administrativa y financiera de la EBC.

Nota: Elaboración propia a partir de resultados de guías de observación.

\section{Conclusiones}

En la actualidad, el mundo globalizado demanda con mayor fuerza programas educativos, cuyo fin sea formar personal altamente capacitado. Los grandes avances y la evolución constante en las tecnologías de información y comunicación ofrecen nuevas alternativas en los procesos evaluativos, permitiendo, con ello, emitir juicios de valor respecto a los objetivos alcanzados por la institución educativa, con base en su función legitimada y formadora por el entorno en el cual se desempeña. Según estos preceptos, los organismos certificadores de la calidad educativa y de los procesos -tanto en el orden nacional e internacional- han desarrollado múltiples modalidades de evaluación de programas educativos con sus criterios y parámetros respectivos.

Con base en lo anterior, se concluye que tanto los programas de Licenciatura en Turismo, ofrecidos por la UVM y por la EBC, presentan deficiencias en cuanto a su organización académico-administrativa. Además, no realizan dentro de sus principales actividades la investigación y generación de nuevos conocimientos. Lo anterior se fundamenta en los resultados obtenidos de la investigación, como fueron:

1) Para la infraestructura se obtuvo que la UVM, respecto al organismo nacional, alcanzó 46 puntos porcentuales y, en lo referente al organismo internacional, fue de 40 puntos porcentuales, lo cual significa un bajo nivel de aspectos para acceder a la certificación. En tanto que la EST logra 91 puntos porcentuales en el nivel nacional y en el interna- 
cional obtuvo 90 puntos porcentuales, con ello se ubica dentro de los parámetros de certificación y, por su parte la EBC, ante CONAET consiguió 53 puntos porcentuales y ante la TedQual, 48 puntos porcentuales, lo que representa un bajo nivel de acreditación en los criterios evaluados.

2) Para la estructura, se encontró que la UVM presenta 32 puntos porcentuales tanto para CONAET como para TedQual, rubro en que se marca como deficiente respecto a los criterios establecidos por los organismos certificadores. En el caso de la EST, alcanzó 89 y 86 puntos porcentuales, lo que representa el acceso a la certificación nacional e internacional, aunque se debe continuar trabajando en este para fortalecerlo; en lo que atañe a la EBC posee 32 y 33 puntos porcentuales, lo que implica que se requiere poner mayor énfasis en este aspecto.

3) Para la supraestructura, se obtuvo que los máximos puntajes logrados por la UVM fueron de 47 y 46 puntos porcentuales respectivamente, resultado que califica su desempeño como no satisfactorio. Por su parte, la EST presenta 92 y 91 puntos porcentuales, lo cual implica el acceso a la certificación nacional e internacional, y por último el caso de la EBC, 51 y 50 puntos porcentuales, da una valoración poco satisfactoria.

De esta forma, se les considera a ambas instituciones sistemas cerrados, ya que, a pesar de encontrarse de manera constante en un estado entrópico, con una homeóstasis negativa y aun cuando tiene recepción de insumos del medio externo, es el único rubro que depende del entorno. Al respecto, los resultados las consideran como autosuficientes.Además, estas dos instituciones, de manera general, no cuentan con el mínimo de criterios requeridos por los organismos certificadores en el orden nacional e internacional.

Caso contrario sucede con la EST del IPN, cuyos resultados obtenidos al interior de la institución, con base en las encuestas aplicadas a profesores, alumnos y administrativos, permitieron evaluarla en forma satisfactoria, pues cuenta con los lineamientos suficientes, se encuentra certificada por CONAET, desde el 31 de agosto de 2006, y con una vigencia de cinco años que culminan el 31 de agosto de 2011, y por TedQual, la cual otorgó la acreditación en diciembre 2007 y cuyo vencimiento expira en diciembre de 2010.

Por tanto, es evidente que toda organización educativa, ante las exigencias impuestas por la globalización económica debe tener acreditados sus programas tanto de licenciatura como de posgrado para ser reconocidos como de calidad ante la sociedad. Ante esta situación, se requiere de trabajo en equipo de la sociedad, del sector productivo, de los cuerpos académicos, de los organismos dedicados a la investigación y de las autoridades locales, regionales y nacionales, mismas que deberán aportar una diversidad de acciones para el mejoramiento de la calidad educativa. Es conveniente no olvidar que toda institución es un sistema con diversos componentes, cuyo principal fin es trabajar colectivamente para lograr la función educativa, proporcionando una formación basada en aspectos humanistas, científicos y tecnológicos, lo que redundará en profesionales mejor preparados para un mundo que cada vez es más exigente y del cual se pueden obtener grandes beneficios, tanto para la institución como para los futuros profesionistas.

Ante esa realidad, el paradigma tradicional de la evaluación se rompe con una metodología innovadora denominada SSM (Soft Systems Methodology), propuesta por Peter Checkland. Esta se orienta hacia la profundización en casos específicos y no a la generalización. Su principal preocupación no solo es medir, sino también cualificar y describir el fenómeno social a partir de rasgos 
determinantes, según sean percibidos por los elementos mismos que están íntimamente relacionados a la problemática estudiada.

\section{Recomendaciones}

Por ello se plantean recomendaciones enfocadas a cambios para la infraestructura, estructura y superestructura, las cuales son tres componentes relevantes en el objeto evaluado con base en la metodología de SSM, los cuales se muestran en las figuras 8, 9 y 10. Además, dichos cambios se clasifican en:

- Cambios estructurales: Son aquellos cambios que se efectúan en aquellas partes de la realidad que, a corto plazo, no cambian; ya que su proceso de adoptar nuevos comportamientos es lento. Estos son: la organización de grupos, estructuras de reporte o estructura de responsabilidad funcional.

- Cambios de procesos: Se efectúan en elementos o realidades dinámicas, pues están continuamente fluyendo en la realidad y modificándose para mejorar o empeorar la situación. Afectan los procesos tanto académicos como administrativos.

- Cambios de actitudes: Son los más cruciales, debido a su condición de intangibles, porque su realización depende de la conciencia individual y colectiva de los seres humanos (Martínez, 2006).

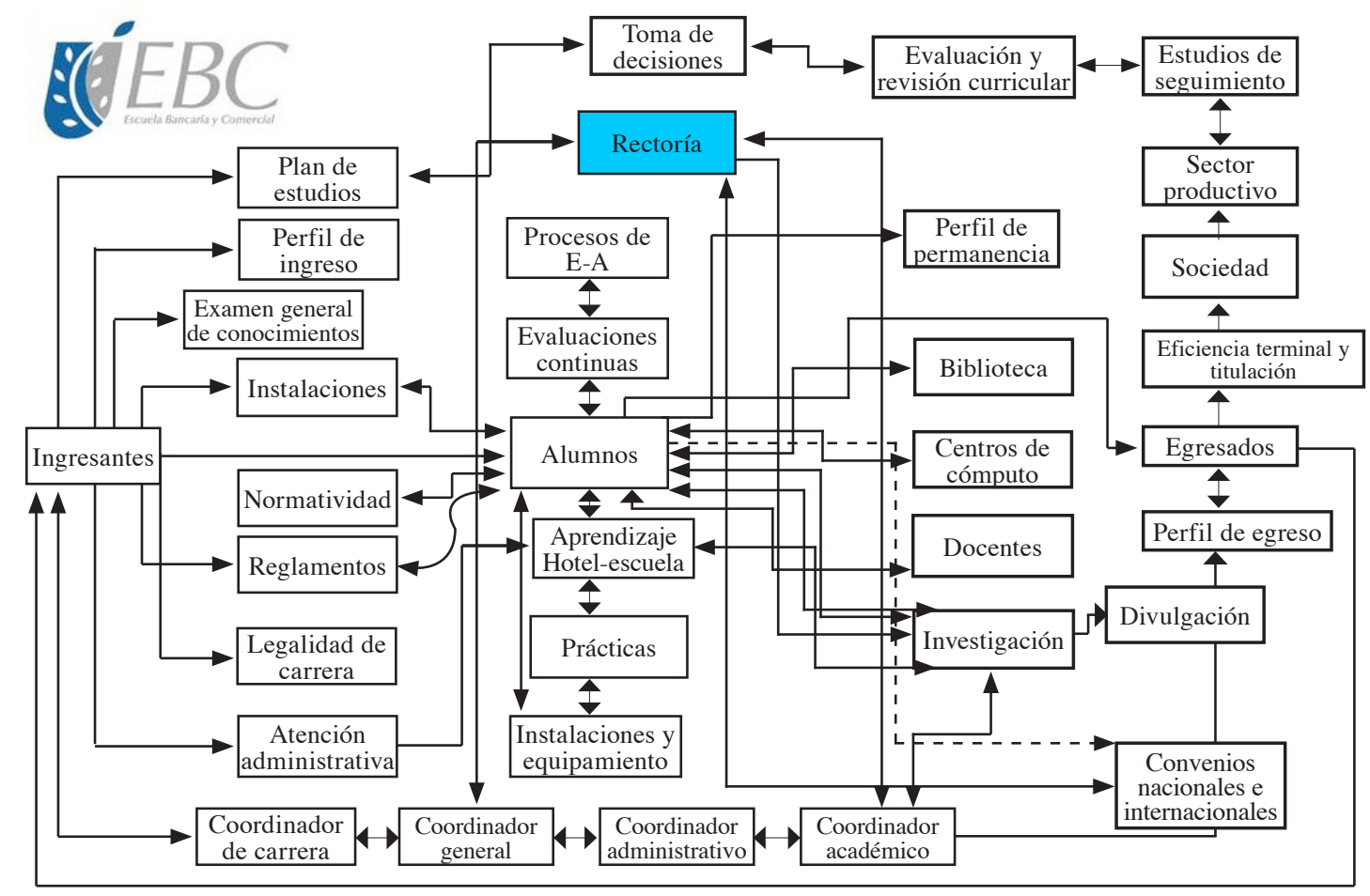

Figura 8. Consideraciones sistémicas para la EBC.

Fuente: Elaboración propia a partir de la metodología de la investigación. 


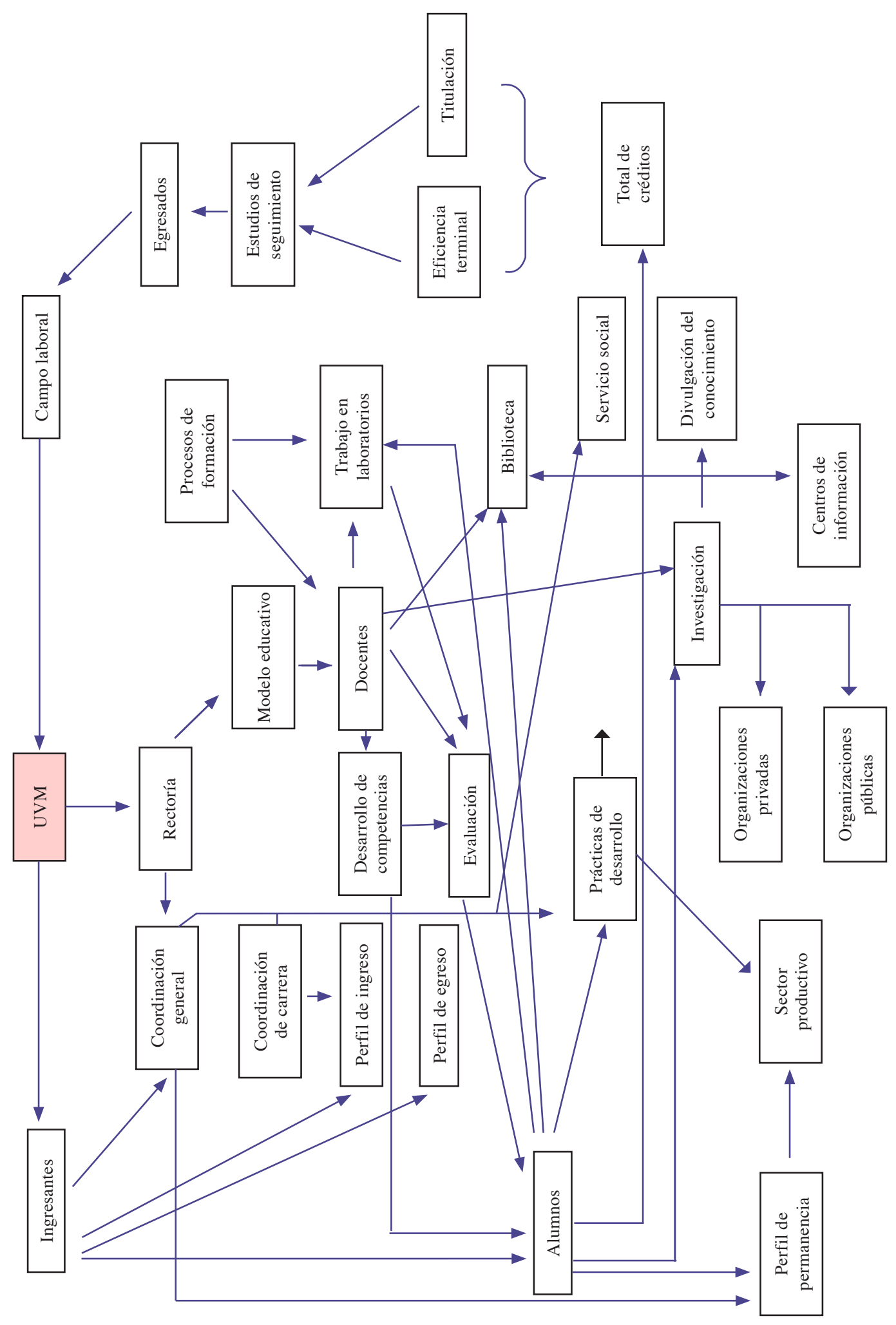

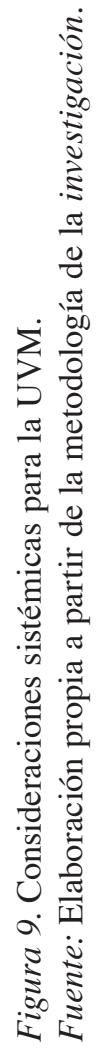









\section{Referencias bibliográficas}

Barranco, J. (2002). Metodología del análisis estructurado de sistemas. España: Universidad Pontificia de Comillas de Madrid.

Cardoso, E., Ramos, J. y Tejeida, R. (2009). Evaluación de los programas educativos desde la perspectiva de los sistemas suaves: Propuesta metodológica. Revista Universidad EAFIT, 45(155), 30-44.

Checkland, P. (2002). La metodología de sistemas suaves en acción. México: Noriega Editores Mega.

Hernández, R., Fernández, C. y Baptista, P. (2002). Metodología de la investigación. México: Mc Graw Hill.

Martínez, A. (2006). Ideas para el cambio y el aprendizaje en la organización: una perspectiva sistémica. Bógota: ECOE.

Zexian, Y. y Xuhui, Y. (2010). A revolution in the field of systems thinking: A review of Checkland's system thinking [Una revolución en el campo del pensamiento sistémico: Una revisión sobre el pensamiento sistémico de Checkland]. Wiley InterScience 2(27), 140-155. doi: 10.1002/ sres. 1021 